\title{
On Fuzzy Soft Linear Operators in Fuzzy Soft Hilbert Spaces
}

\author{
Nashat Faried $\mathbb{D},{ }^{1}$ Mohamed S. S. Ali $\mathbb{D}^{2},{ }^{2}$ and Hanan H. Sakr $\mathbb{D}^{2}$ \\ ${ }^{1}$ Department of Mathematics, Faculty of Science, Ain Shams University, 11566 Cairo, Egypt \\ ${ }^{2}$ Department of Mathematics, Faculty of Education, Ain Shams University, 11341 Cairo, Egypt
}

Correspondence should be addressed to Hanan H. Sakr; henna_66@yahoo.com

Received 24 January 2020; Accepted 9 May 2020; Published 31 July 2020

Academic Editor: Micah Osilike

Copyright (C) 2020 Nashat Faried et al. This is an open access article distributed under the Creative Commons Attribution License, which permits unrestricted use, distribution, and reproduction in any medium, provided the original work is properly cited.

Due to the difficulty of representing problem parameters fuzziness using the soft set theory, the fuzzy soft set is regarded to be more general and flexible than using the soft set. In this paper, we define the fuzzy soft linear operator $\tilde{T}$ in the fuzzy soft Hilbert space $\tilde{H}$ based on the definition of the fuzzy soft inner product space $(\tilde{U}, \widetilde{\langle\cdot, \cdot\rangle})$ in terms of the fuzzy soft vector $\tilde{v}_{f_{G(e)}}$ modified in our work. Moreover, it is shown that $\mathbb{C}^{n}(A), \mathbb{R}^{n}(A)$ and $\ell_{2}(A)$ are suitable examples of fuzzy soft Hilbert spaces and also some related examples, properties and results of fuzzy soft linear operators are introduced with proofs. In addition, we present the definition of the fuzzy soft orthogonal family and the fuzzy soft orthonormal family and introduce examples satisfying them. Furthermore, the fuzzy soft resolvent set, the fuzzy soft spectral radius, the fuzzy soft spectrum with its different types of fuzzy soft linear operators and the relations between those types are introduced. Moreover, the fuzzy soft right shift operator and the fuzzy soft left shift operator are defined with an example of each type on $\ell_{2}(A)$. In addition, it is proved, on $\ell_{2}(A)$, that the fuzzy soft point spectrum of fuzzy soft right shift operator has no fuzzy soft eigenvalues, the fuzzy soft residual spectrum of fuzzy soft right shift operator is equal to the fuzzy soft comparison spectrum of it and the fuzzy soft point spectrum of fuzzy soft left shift operator is the fuzzy soft open disk $|\tilde{\lambda}| \tilde{<} \tilde{1}$. Finally, it is shown that the fuzzy soft Hilbert space is fuzzy soft self-dual in this generalized setting.

\section{Introduction}

In real world, the complexity generally arises from uncertainty in the form of ambiguity. So, we always have many complicated problems in the areas like economics, engineering, medical science, environmental science, sociology, business management and many other fields. We cannot successfully use classical mathematical methods to overcome difficulties of uncertainties in those problems.

In 1965, Zadeh [1] proposed an extension of the set theory which is the theory of fuzzy sets to deal with uncertainty. Just as a crisp set on a universal set $X$ is defined by its characteristic function from $X$ to $\{0,1\}$, a fuzzy set on a domain $X$ is defined by its membership (characteristic) function from $X$ to $[0,1]$.

In 1999, Molodtsov [2] introduced an extension of the set theory namely soft set theory to overcome uncertainties and solve complicated problems which cannot be dealt with by classical methods in many areas such as Riemann integra- tion, measure theory, environmental science, decision-making, game theory, physics, engineering, computer science, medicine, economics and many other fields. The soft set is a mathematical tool for modeling uncertainty by associating a set with a set of parameters, i.e., it is a parameterized family of subsets of the universal set. After that, many researchers introduced new extended concepts based on soft sets, gave examples for them and studied their properties like soft point [3], soft metric spaces [4], soft normed spaces [5], soft inner product spaces [6] and soft Hilbert spaces [7], etc. In addition, many researchers presented some applications of soft set theory in different areas (see [8-12]).

But almost all the time, although this progress, in real life problems and situations, we still have inexact information about our considered objects. So, to improve those two concepts; fuzzy set and soft set, Maji et al. [13] combined them together in one concept and called this new concept fuzzy soft set. This new concept widened the soft sets approach from crisp (ordinary) cases to fuzzy cases which is more 
general than any other. In recent years, many researchers applied this notion and gave some concepts such as fuzzy soft point [14], fuzzy soft real number [15], fuzzy soft metric spaces [16] and fuzzy soft normed spaces [17]. In addition, many researchers presented some applications of fuzzy soft set theory in different areas (see [18-25]).

In this work, we progress on these stated previous studies by introducing the fuzzy soft inner product on fuzzy soft vector spaces, the fuzzy soft Cauchy-Schwartz inequality, the fuzzy soft orthogonality and the fuzzy soft Hilbert spaces. Moreover, we define the fuzzy soft linear operators in fuzzy soft Hilbert spaces, establish their related theorems, introduce fuzzy soft spectral theory of them and prove the fuzzy soft self-duality of fuzzy soft Hilbert spaces. The rest of the paper is organized as the following. Section 2 introduces the basic needed concepts and definitions. Section 3 studies the fuzzy soft linear operators in fuzzy soft Hilbert spaces and some related examples and properties. Furthermore, in section 3, fuzzy soft right shift operator, fuzzy soft left shift operator, fuzzy soft resolvent set, fuzzy soft spectral radius, fuzzy soft spectrum with its different types of them are investigated. Finally, in section 3, we show that the fuzzy soft Hilbert space is fuzzy soft self-dual. Section 4 provides conclusions and open questions for further investigations.

\section{Definitions and Preliminaries}

The aim of this section is to list some notations, definitions and preliminaries for fuzzy set, soft set and fuzzy soft set needed in the following discussion. In addition, it presents the fuzzy soft point definition including our present modification.

Definition 1 (Fuzzy set) [1]. Let U be a universal set (space of points or objects). A fuzzy set (class) $X$ over $U$ is a set characterized by a function $f_{X}: U \rightarrow[0,1] . f_{X}$ is called the membership, characteristic or indicator function of the fuzzy set $X$ and the value $f_{X}(u)$ is called the grade of membership of $u$ $\in U$ in $X$.

Definition 2 (Soft set) $([2,26])$. A pair $(U, E)$ is said to be a soft set over a non-empty set $X$ provided that $U$ is a mapping of a set of parameters $E$ into $2^{U}$. A soft set is identified as a set of ordered pairs: $(U, E)=\left\{(e, U(e)): e \in E\right.$ and $\left.G(e) \in 2^{U}\right\}$.

Example 3. Consider a soft set $(F, A)$ which describes "attractiveness of markets" under the consideration of a decision maker to purchase. Suppose that there are five markets to be considered in the universal set $U$, denoted by $U=\left\{m_{1}\right.$, $\left.m_{2}, m_{3}, m_{4}, m_{5}\right\}$ and $A=\left\{a_{1}, a_{2}, a_{3}\right\}$, where $a_{i}(i=1,2,3)$ stands for the parameters in a word of "luxurious", "expensive", and "in a suitable location", respectively. Thus, to define a soft set means to represent luxurious markets, expensive markets and so on. We can write the soft set $(F, A)$ over $U$ by the relation $(F, A)=\left\{\left(a_{1},\left\{m_{2}\right.\right.\right.$, $\left.\left.\left.m_{4}\right\}\right),\left(a_{2},\left\{m_{5}\right\}\right),\left(a_{3},\left\{m_{1}, m_{3}, m_{4}\right\}\right)\right\}$. This soft set can be represented in Table 1 .
TABLE 1: Representation of the soft set $(F, A)$.

\begin{tabular}{llll}
\hline$U$ & $a_{1}$ & $a_{2}$ & $a_{3}$ \\
\hline$m_{1}$ & 0 & 0 & 1 \\
$m_{2}$ & 1 & 0 & 0 \\
$m_{3}$ & 0 & 0 & 1 \\
$m_{4}$ & 1 & 0 & 1 \\
$m_{5}$ & 0 & 1 & 0 \\
\hline
\end{tabular}

TABLE 2: Representation of the fuzzy soft set $(G, A)$.

\begin{tabular}{lccc}
\hline$U$ & $a_{1}$ & $a_{2}$ & $a_{3}$ \\
\hline$m_{1}$ & 0.1 & 0 & 1 \\
$m_{2}$ & 0.6 & 0 & 0 \\
$m_{3}$ & 0 & 0 & 0.7 \\
$m_{4}$ & 0.8 & 0.2 & 0.9 \\
$m_{5}$ & 0.3 & 0.8 & 0 \\
\hline
\end{tabular}

Definition 4 (Fuzzy soft set) [13]. Let $U$ be a universal set, $E$ be a set of parameters and $A \subseteq E$. A pair $(G, A)$ is called a fuzzy soft set over $U$, where $G$ is a mapping given by $G$ $: A \longrightarrow \mathscr{F}(U), \mathscr{F}(U)$ is the family of all fuzzy subsets of $U$ (the power set of fuzzy sets on $U$ ) and the fuzzy subset of $U$ is defined as a map $f$ from $U$ to $[0,1]$. The family of all fuzzy soft sets $(G, A)$ over a universal set $U$, in which all the parameter sets $A$ are the same, is denoted by FSS $(U)_{A}=\operatorname{FSS}(\tilde{U})$.

Example 5. Example (3) can be characterized by a membership function instead of 0,1 numbers which associates each element with a real number in the interval $[0,1]$, then we can write the fuzzy soft set $(G, A)$ over $U$ as $(G, A)=\left\{G\left(a_{1}\right)\right.$ $=\left\{\left(m_{1}, 0.1\right),\left(m_{2}, 0.6\right),\left(m_{4}, 0.8\right),\left(m_{5}, 0.3\right)\right\}, G\left(a_{2}\right)=\left\{\left(m_{4}\right.\right.$, $\left.0.2),\left(m_{5}, 0.8\right)\right\}, G\left(a_{3}\right)=\left\{\left(m_{1}, 1\right),\left(m_{3}, 0.7\right),\left(m_{4}, 0.9\right)\right\}$. This fuzzy soft set can be represented in Table 2.

The following definition and its consequent related definitions take their present formula according to our modification as follows:

Definition 6 (Fuzzy soft point) [14]. The fuzzy soft set $(G$, $A) \in \operatorname{FSS}(\tilde{U})$ is called a fuzzy soft point over $U$, denoted by $\left(u_{f_{G(e)}}, A\right)$ (briefly denoted by $\left.\tilde{u}_{f_{G(e)}}\right)$, if for the element $e \in A$ and $u \in U(\alpha \in(0,1]$ is the value of the membership degree),

$$
f_{G(e)}(u)=\left\{\begin{array}{l}
\alpha, \text { if } u=u_{0} \in U \text { and } e=e_{0} \in A, \\
0, \text { if } u \in U-\left\{u_{0}\right\} \text { or } e \in A-\left\{e_{0}\right\}
\end{array} .\right.
$$

The fuzzy soft point can be considered as the quadruple $\left(u_{0}, e_{0}, G, \alpha\right)$.

Example 7. Suppose that there are four points in the universal set $U$, i.e., $U=\left\{u^{1}, u^{2}, u^{3}, u^{4}\right\}$ and let $A=\left\{e_{1}, e_{2}, e_{3}, e_{4}, e_{5}\right\}$ 
$\subseteq E$ be the set of parameters. Then, $\left(u^{2}, e_{5}, G, 0.6\right)=\tilde{u}_{0.6_{G\left(e_{5}\right)}}$ is a fuzzy soft point over $U$.

Definition 8 (The complement of a fuzzy soft point) [27]. The fuzzy soft point $\tilde{u}_{f_{G} C_{(e)}}$ is called the fuzzy soft complement of a fuzzy soft point $\tilde{u}_{f_{G(e)}}$, denoted by $\left(\tilde{u}_{f_{G(e)}}\right)^{C}$, if for the element $e \in A$ and $u \in U$,

$$
f_{G^{C}(e)}(u)=\left\{\begin{array}{l}
1-f_{G(e)}(u), \text { if } u=u_{0} \in U \text { and } e=e_{0} \in A \\
0, \text { if } u \in U-\left\{u_{0}\right\} \text { or } e \in A-\left\{e_{0}\right\}
\end{array}\right.
$$

Example 9. The complement of the fuzzy soft point stated in Example (7) is the fuzzy soft point $\left(u^{2}, e_{5}, G, 0.4\right)=\tilde{u}_{0.4_{G\left(e_{5}\right)}^{2}}$.

The collection of all fuzzy soft points over $U$ is denoted by $\operatorname{FSP}(U)_{A}=\operatorname{FSP}(\tilde{U}) \cdot \mathbb{R}(A)$ denotes the set of all fuzzy soft real numbers and $\mathbb{R}^{+}(A)$ denotes the set of all non-negative fuzzy soft real numbers (such as $\tilde{r}$, in symbol). $\mathbb{C}(A)$ denotes the set of all fuzzy soft complex numbers (such as $\tilde{c}$, in symbol). Note that the fuzzy soft zero vector $\tilde{\theta}=(\tilde{0}, \tilde{0}, \tilde{0}, \tilde{0})$ and the fuzzy soft unit vector $\tilde{j}=(\tilde{1}, \tilde{1}, \tilde{1}, \tilde{1})$.

Definition 10 (Fuzzy soft vector space)([17]). Let $\mathrm{U}$ be a vector space over a field $\mathbb{K}(\mathbb{K}=\mathbb{R})$ and the parameter set $\mathrm{E}$ be the set of all real numbers $\mathbb{R}$ and $A \subseteq E$. The fuzzy soft set $(G, A) \in \operatorname{FSS}(\tilde{U})$ is called a fuzzy soft vector over $U$, denoted by $\left(v_{f_{G(e)}}, A\right)$ (briefly denoted by $\left.\tilde{v}_{f_{G(e)}}\right)$, if there is exactly one $e \in A$ such that $f_{G(e)}(v)=\alpha$ for some $v \in U$ and $\left.f_{G(e}{ }^{\prime}\right)(v)=0$ for all $e^{\prime} \in A-\{e\} \quad(\alpha \in(0,1]$ is the value of the membership degree). The set of all fuzzy soft vectors over $U$ is denoted by $\operatorname{FSV}(U)_{A}=F S V(\tilde{U})$. The set $\tilde{V} \tilde{=} F S V(\tilde{U})$ is said to be a fuzzy soft vector space or a fuzzy soft linear space of $U$ over $\mathbb{K}$ if $\tilde{V}(e)$ is a vector subspace of $U$, for all $e \in A$. $\tilde{V}$ is a fuzzy soft vector space according to the following two operations:

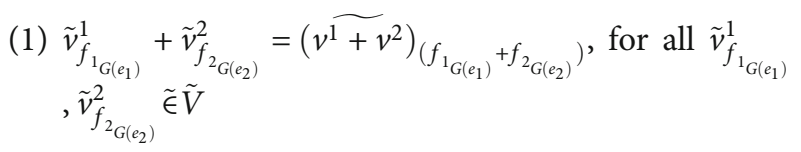

(2) $\tilde{r} \cdot \tilde{v}_{f_{G(e)}}=(\tilde{r v})_{f_{G(r e)}}$, for all $\tilde{v}_{f_{G(e)}} \tilde{\epsilon} \tilde{V}$ and for all $\tilde{r} \tilde{\epsilon} \mathbb{R}(A)$.

Example 11. Consider the Euclidean $n$ - dimensional space $\mathbb{R}^{n}$ over $\mathbb{R}$.

Let $A=\{1,2,3, \cdots, n\}$ be the set of parameters. Let $\tilde{V}: A$ $\longrightarrow \mathscr{F}\left(\mathbb{R}^{n}\right)$ be defined as follows:

$\tilde{V}(i)=\left\{\tilde{v}_{f_{n_{G\left(e_{n}\right)}}^{n}} \tilde{\in} \mathbb{R}^{n}(A) ; i^{t h}\right.$ coordinate of $\tilde{v}_{f_{n_{G\left(e_{n}\right)}}^{n}}$ is $\left.\tilde{\theta}\right\}, i=$ $1,2, \cdots, n$.

Then $\tilde{V}$ is a fuzzy soft vector space or a fuzzy soft linear space of $\mathbb{R}^{n}$ over $\mathbb{R}$.

In addition, Let $\tilde{v}_{f_{n_{G\left(e_{n}\right)}}^{n}}$ be a fuzzy soft element of $\tilde{V}$ as follows:

$$
\tilde{v}_{f_{n_{G\left(e_{n}\right)}}^{n}}=\left(\tilde{j}, \tilde{j}, \cdots, \tilde{j}, \tilde{\theta}_{\left(i^{t h}\right)}, \tilde{j}, \cdots, \tilde{j}\right) \tilde{\epsilon} \mathbb{R}^{n}(A), \quad i=1,2, \cdots, n .
$$

Then $\tilde{v}_{f_{n_{G\left(e_{n}\right)}}^{n}}$ is a fuzzy soft vector of $\tilde{V}$.

Definition 12 (Fuzzy soft metric space) [16]. A fuzzy soft metric space $(\tilde{U}, \tilde{d})$ is a fuzzy soft set $\tilde{U}$ with a fuzzy soft realvalued function $\tilde{d}: \operatorname{FSP}(\tilde{U}) \times F S P(\tilde{U}) \longrightarrow \mathbb{R}^{+}(A)$ satisfying the fuzzy soft metric conditions as the following:

(FSM1) $\tilde{d}\left(\tilde{u}_{f_{1_{G\left(e_{1}\right)}}^{1}}, \tilde{u}_{f_{2_{G\left(e_{2}\right)}}^{2}}\right) \tilde{\geq} \tilde{0}$, for all $\tilde{u}_{f_{1_{G\left(e_{1}\right)}}}^{1}, \tilde{u}_{f_{2_{G\left(e_{2}\right)}}^{2}} \in \mathcal{\epsilon} P($ $\tilde{U})$, and $\tilde{d}\left(\tilde{u}_{f_{1_{G\left(e_{1}\right)}}^{1}}, \tilde{u}_{\left.f_{2_{G\left(e_{2}\right)}}^{2}\right)}^{2} \tilde{=} \tilde{0} \Longleftrightarrow \tilde{u}_{f_{1_{G\left(e_{1}\right)}}}^{1} \tilde{u}_{f_{2_{G\left(e_{2}\right)}}^{2}}^{2}\right.$.

(FSM2) $\quad \tilde{d}\left(\tilde{u}_{f_{1_{G\left(e_{1}\right)}}^{1}}^{1}, \tilde{u}_{f_{2_{G\left(e_{2}\right)}}^{2}}\right) \tilde{=} \tilde{d}\left(\tilde{u}_{f^{2_{G\left(e_{2}\right)}}}^{2}, \tilde{u}_{f_{1_{G\left(e_{1}\right)}}^{1}}\right), \quad$ for all

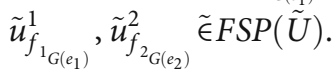

(FSM3) $\tilde{d}\left(\tilde{u}_{f_{1_{G\left(e_{1}\right)}}^{1}}, \tilde{u}_{f_{2_{G\left(e_{2}\right)}}}^{2}\right) \tilde{\leq} \tilde{d}\left(\tilde{u}_{f_{1_{G\left(e_{1}\right)}}^{1}}^{1}, \tilde{u}_{f^{3_{G\left(e_{3}\right)}}}^{3}\right)+\tilde{d}\left(\tilde{u}_{f_{3_{G\left(e_{3}\right)}}^{3}}^{3}\right.$, $\left.\tilde{u}_{f_{2_{G\left(e_{2}\right)}}^{2}}^{2}\right)$, for all $\tilde{u}_{f_{1_{G\left(e_{1}\right)}}^{1}}^{1}, \tilde{u}_{f_{2_{G\left(e_{2}\right)}}^{2}}^{2}, \tilde{u}_{f_{3_{G\left(e_{3}\right)}}} \tilde{\epsilon} F P(\tilde{U})$.

Definition 13 (Fuzzy soft normed space) [17]. Let $F S V(\tilde{U})$ be a fuzzy soft vector space. Then, a mapping $\widetilde{\|\cdot\|}: F S V(\tilde{U})$ $\longrightarrow \mathbb{R}^{+}(A)$ is said to be a fuzzy soft norm on $\operatorname{FSV}(\tilde{U})$ if $\widetilde{\|\cdot\|}$ satisfies the following conditions:

(FSN1) $\left\|\widetilde{\tilde{v}_{f_{G(e)}}}\right\| \tilde{\geq} \tilde{0}$, for all $\tilde{v}_{f_{G(e)}} \tilde{\epsilon} F S V(\tilde{U})$, and $\left\|\widetilde{\tilde{v}_{f_{G(e)}}}\right\| \tilde{=} \tilde{0}$ $\Longleftrightarrow \tilde{v}_{f_{G(e)}} \tilde{=} \tilde{\theta}$.

(FSN2) $\left\|\tilde{r} \cdot \widetilde{\tilde{v}_{f_{G(e)}}}\right\| \tilde{=}|\tilde{r}|\left\|\widetilde{\tilde{v}_{f_{G(e)}}}\right\|$, for all $\tilde{v}_{f_{G(e)}} \tilde{\epsilon} F S V(\tilde{U})$ and for all fuzzy soft scalar $\tilde{r}$.

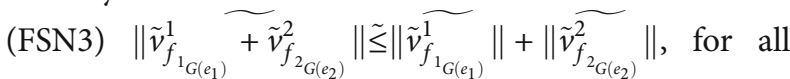
$\tilde{v}_{f_{1_{G\left(e_{1}\right)}}^{1}}, \tilde{v}_{f_{2_{G\left(e_{2}\right)}}}^{2} \tilde{\epsilon} F S V(\tilde{U})$.

\section{Main Results}

The aim of this section is to introduce the fuzzy soft inner product spaces, the fuzzy soft Hilbert spaces and the fuzzy soft linear operators in fuzzy soft Hilbert spaces and to study some theorems and results of them. In addition, fuzzy soft resolvent set, fuzzy soft spectral radius, fuzzy soft spectrum with its different types of them and more results are established. Furthermore, fuzzy soft right shift operator and fuzzy soft left shift operator are defined. Finally, the fuzzy soft selfduality of fuzzy soft Hilbert space is introduced.

Definition 14 (Fuzzy soft inner product space) [28]. Let FS $V(\tilde{U})$ be a fuzzy soft vector space. Then, the mapping $\widetilde{\langle\cdot, \cdot\rangle}$ $: F S V(\tilde{U}) \times F S V(\tilde{U}) \longrightarrow \mathbb{C}(A)$ is said to be a complex fuzzy soft inner product (shortly, fuzzy soft inner product) on $F S$ $V(\tilde{U})$ if $\langle\cdot, \cdot\rangle$ satisfies the following axioms:

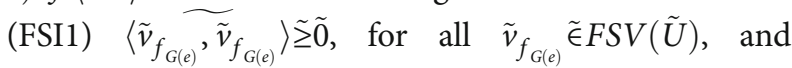

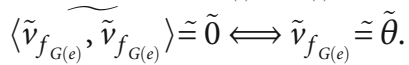




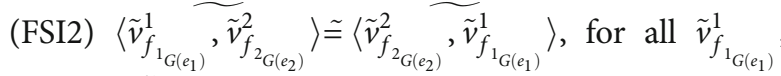
$\tilde{v}_{f_{2_{G\left(e_{2}\right)}}^{2}}^{2} \tilde{\epsilon} F S(\tilde{U})$, where bar denotes the complex conjugate of the fuzzy soft complex number.

(FSI3) $\left\langle\tilde{c} \cdot \tilde{v}_{f_{1_{G\left(e_{1}\right)}}^{1}}^{1}, \tilde{v}_{f_{2_{G\left(e_{2}\right)}}^{2}}^{2}\right\rangle=\tilde{c}\left\langle\tilde{v}_{f_{1_{G\left(e_{1}\right)}}^{1}}^{1}, \tilde{v}_{f_{{ }_{G}\left(e_{2}\right)}^{2}}^{2}\right\rangle, \quad$ for all $\tilde{v}_{f_{1_{G\left(e_{1}\right)}}}^{1}, \tilde{v}_{f_{\mathrm{G}_{G\left(e_{2}\right)}}^{2}}^{2} \tilde{\epsilon} F S V(\tilde{U})$ and for all fuzzy soft scalar $\tilde{c}$.

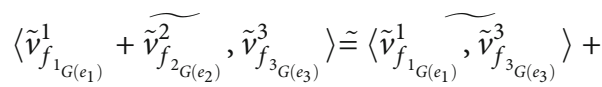

$\left\langle\tilde{v}_{f_{G\left(e_{2}\right)}}^{2}, \tilde{v}_{f^{3 G\left(e_{3}\right)}}^{3}\right\rangle$, for all $\tilde{v}_{f_{1_{G\left(e_{1}\right)}}^{1}}^{1}, \tilde{v}_{f_{2_{G\left(e_{2}\right)}}^{2}}, \tilde{v}_{f^{3_{G\left(e_{3}\right)}}}^{3} \quad \tilde{\epsilon} F S V(\tilde{U})$.

The fuzzy soft vector space $F S V(\tilde{U})$ with a fuzzy soft inner product $\langle\cdot \widetilde{\cdot, \cdot}\rangle$ is said to be a complex fuzzy soft inner product space (shortly, fuzzy soft inner product space) and is denoted by $(\tilde{U}, \widetilde{\langle\cdot, \cdot\rangle})$.

Definition 15 (Real fuzzy soft inner product space) [28]. If the mapping $\widetilde{\langle\cdot, \cdot\rangle}$ in the above Definition (14) is replaced by $\widetilde{\langle\cdot, \cdot\rangle}: F S V(\tilde{U}) \times F S V(\tilde{U}) \longrightarrow \mathbb{R}(A)$, then it is called a real fuzzy soft inner product space and its Conditions (Axioms (FSI1),(FSI3),(FSI4)) are the same, but the Condition (FSI2) is replaced by

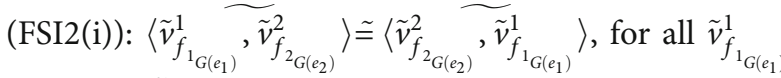

$$
\begin{aligned}
& \text {, } \tilde{v}_{f_{G\left(e_{2}\right)}}^{2} \tilde{\epsilon} F S V(\tilde{U}) \text {. }
\end{aligned}
$$

Theorem 16 (Fuzzy soft Cauchy-Schwartz inequality) [28]. Let $(\tilde{U}, \widetilde{\langle\cdot, \cdot\rangle})$ be a fuzzy soft inner product space, then for all $\tilde{v}_{f_{l_{G\left(e_{1}\right)}}^{1}}^{1}, \tilde{v}_{f_{2_{G\left(e_{2}\right)}}^{2}}^{2} \tilde{\epsilon} \operatorname{FV}(\tilde{U})$, we have

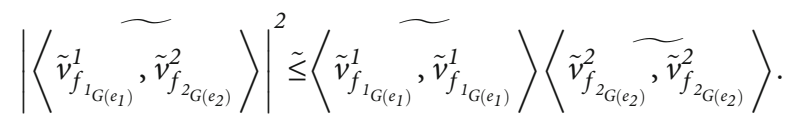

Theorem 17 (see [28]). For all $\tilde{v}_{f_{G(e)}} \tilde{\epsilon} F S V(\tilde{U})$, a fuzzy soft inner product space $(\tilde{U}, \widetilde{\langle\cdot, \cdot\rangle})$ can be considered as a fuzzy soft normed space with $\left\|\widetilde{\tilde{v}_{f_{G(e)}}}\right\| \simeq \sqrt{\left\langle\tilde{v}_{f_{G(e)}, \widetilde{v_{f}}}\right\rangle}$.

Example 18. $\mathbb{C}^{n}(A)$ the fuzzy soft complex Euclidean space (the space of all fuzzy soft $n$ - dimensional complex numbers) is a complex fuzzy soft inner product space with the complex fuzzy soft inner product defined as follows:

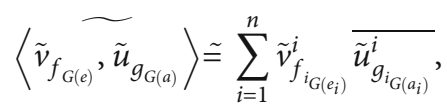

for all $\tilde{v}_{f_{G(e)}}, \tilde{u}_{g_{G(a)}} \tilde{\epsilon} \mathbb{C}^{n}(A)$.

Solution. The proof is straightforward by applying the conditions of the complex fuzzy soft inner product space stated in Definition (14).
Example 19. $\mathbb{R}^{n}(A)$ the fuzzy soft real Euclidean space (the space of all fuzzy soft $n$ - dimensional real numbers) is a real fuzzy soft inner product space with the real fuzzy soft inner product defined as follows:

$$
\left\langle\tilde{v}_{f_{G(e)}} \widetilde{\tilde{u}_{g_{G(a)}}}\right\rangle \tilde{=} \sum_{i=1}^{n} \tilde{v}_{f_{i_{G\left(e_{i}\right)}}^{i}} \overline{\tilde{u}_{g^{i} G\left(a_{i}\right)}^{i}},
$$

for all $\tilde{v}_{f_{G(e)}}, \tilde{u}_{g_{G(a)}} \tilde{\epsilon} \mathbb{R}^{n}(A)$.

Solution. The proof is straightforward by applying the conditions of the real fuzzy soft inner product space stated in Definition (15).

Example 20. $\ell_{2}(A)$ the space of all fuzzy soft squaresummable sequences is a complex fuzzy soft inner product space with the complex fuzzy soft inner product defined as follows:

$$
\left\langle\tilde{v}_{f_{G(e)}} \widetilde{\tilde{u}_{g_{G(a)}}}\right\rangle \tilde{=} \sum_{i=1}^{\infty} \tilde{v}_{f_{i_{G\left(e_{i}\right)}}^{i}} \overline{\tilde{u}_{g_{i_{G\left(a_{i}\right)}}^{i}}}
$$

for all $\tilde{v}_{f_{G(e)}}, \tilde{u}_{g_{G(a)}} \tilde{\epsilon} \ell_{2}(A)$.

Solution. The proof is straightforward by applying the conditions of the complex fuzzy soft inner product space stated in Definition (14).

Example 21. $\mathscr{C}_{[\tilde{0}, \tilde{1}]}(A)$ the space of all fuzzy soft complexvalued continuous functions on $[\tilde{0}, \tilde{1}]$ is a complex fuzzy soft inner product space with the complex fuzzy soft inner product defined as follows:

$$
\left\langle\tilde{\eta}_{f_{G(e)}} \widetilde{\tilde{\xi}_{g_{G(a)}}}\right\rangle \tilde{=} \int_{i=\tilde{0}}^{\tilde{1}} \tilde{\eta}_{f_{i_{G\left(e_{i}\right)}}^{i}}(\tilde{x}) \overline{\tilde{\xi}_{g_{i_{G\left(a_{i}\right)}}^{i}}(\tilde{x})} d \tilde{x},
$$

for all $\tilde{\eta}_{f_{G(e)}}, \tilde{\xi}_{g_{G(a)}} \in \mathscr{C}_{[\tilde{0}, \tilde{1}]}(A)$.

Solution. The proof is straightforward by applying the conditions of the complex fuzzy soft inner product space stated in Definition (14).

Theorem 22 (Fuzzy soft polarization identity) [28]. Let ( $\tilde{U}$, $\widetilde{\langle\cdot, \cdot\rangle)}$ be a complex fuzzy soft inner product space and let $\tilde{v}_{f_{1_{G\left(e_{1}\right)}}^{1}}, \tilde{v}_{f_{2_{G\left(e_{2}\right)}}^{2}}^{2} \tilde{\epsilon} S V(\tilde{U})$. Then, we can write the fuzzy soft 
polarization identity in the following formula:

$$
\begin{aligned}
& \left\langle\tilde{v}_{f_{I_{G\left(e_{1}\right)}}^{1}}, \tilde{v}_{f_{{ }_{G\left(e_{2}\right)}}^{2}}^{2}\right\rangle \tilde{=} \frac{\tilde{1}}{\tilde{4}}\left(\left\|\tilde{v}_{f_{I_{G\left(e_{I}\right)}}^{1}} \underset{\tilde{v}_{f_{2_{G\left(e_{2}\right)}}}^{2}}{\|^{2}}\right\|^{2}\right.
\end{aligned}
$$

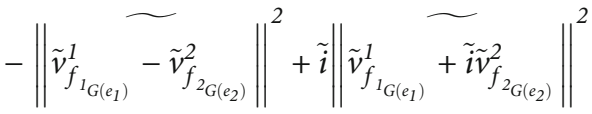

$$
\begin{aligned}
& \left.-\tilde{i}\left\|\tilde{v}_{f_{I_{G\left(e_{1}\right)}}^{1}}-\tilde{i}_{\tilde{v}_{f_{G\left(e_{2}\right)}}^{2}}^{2}\right\|^{2}\right)
\end{aligned}
$$

for all $\tilde{v}_{f_{l_{G\left(e_{1}\right)}}^{1}}^{1}, \tilde{v}_{f_{2_{G\left(e_{2}\right)}}^{2}}^{2} \tilde{\epsilon} \operatorname{FS}(\tilde{U})$.

Definition 23 (Fuzzy soft Hilbert space) [29]. Let $(\tilde{U}, \widetilde{\langle\cdot, \cdot\rangle})$ be a fuzzy soft inner product space. Then, this space, which is fuzzy soft complete in the induced fuzzy soft norm stated in Theorem (17), is called a fuzzy soft Hilbert space, denoted by $(\tilde{H}, \widetilde{\langle\cdot, \cdot\rangle})$ (shortly $\tilde{H}$ ). It is clear that every fuzzy soft Hilbert space is a fuzzy soft Banach space.

Example 24. The space $\mathbb{C}^{n}(A)$ the fuzzy soft complex Euclidean space (the space of all fuzzy soft $n$ - dimensional complex numbers) is a complex fuzzy soft Hilbert space with the complex fuzzy soft inner product defined as follows:

$$
\left\langle\tilde{v}_{f_{G(e)},}, \tilde{u}_{g_{G(a)}}\right\rangle \tilde{=} \sum_{i=1}^{n} \tilde{v}_{f_{i_{G\left(e_{i}\right)}}^{i}} \overline{\tilde{u}_{g_{i_{G\left(a_{i}\right)}}^{i}}},
$$

for all $\tilde{v}_{f_{G(e)}}, \tilde{u}_{g_{G(a)}} \tilde{\epsilon} \mathbb{C}^{n}(A)$.

Solution. We have $\mathbb{C}^{n}(A)$ is an fuzzy soft inner product space from Example (18). Using Theorem (17), we obtain:

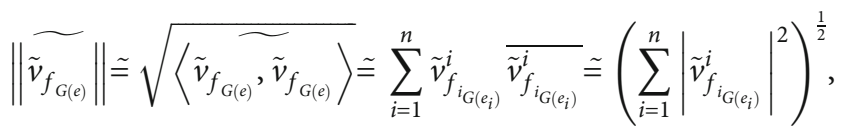

and since $\mathbb{C}^{n}(A)$ is fuzzy soft complete in this fuzzy soft norm. Then, $\mathbb{C}^{n}(A)$ is an fuzzy soft complete fuzzy soft inner product space, i.e., an fuzzy soft Hilbert space.

Example 25. The space $\mathbb{R}^{n}(A)$ the fuzzy soft real Euclidean space (the space of all fuzzy soft $n$ - dimensional real numbers) is an fuzzy soft Hilbert space with the real fuzzy soft inner product defined as follows:

$$
\left\langle\tilde{v}_{f_{G(e)},}, \tilde{u}_{g_{G(a)}}\right\rangle \tilde{=} \sum_{i=1}^{n} \tilde{v}_{f_{i_{G\left(e_{i}\right)}}^{i}} \overline{\tilde{u}_{g_{i_{G\left(a_{i}\right)}}^{i}}},
$$

for all $\tilde{v}_{f_{G(e)}}, \tilde{u}_{g_{G(a)}} \tilde{\epsilon} \mathbb{R}^{n}(A)$.

Solution. The proof is easy by using Example (19) and Theorem (17), similarly as Example (24).
Example 26. The space $\ell_{2}(A)$ the space of all fuzzy soft square-summable sequences is an fuzzy soft Hilbert space with the complex fuzzy soft inner product defined as follows:

$$
\left\langle\tilde{v}_{f_{G(e)}} \widetilde{\tilde{u}_{g_{G(a)}}}\right\rangle \stackrel{=}{=} \sum_{i=1}^{\infty} \tilde{v}_{f_{i_{G\left(e_{i}\right)}}^{i}} \overline{\tilde{u}_{g^{i} G\left(a_{i}\right)}^{i}},
$$

for all $\tilde{v}_{f_{G(e)}}, \tilde{u}_{g_{G(a)}} \tilde{\epsilon} \ell_{2}(A)$.

Solution. The space $\ell_{2}(A)$ is fuzzy soft complete as an fuzzy soft normed space. But $\ell_{2}(A)$ is an fuzzy soft inner product space from Example (20). Then, $\ell_{2}(A)$ is an fuzzy soft Hilbert space.

Definition 27 (Fuzzy soft orthogonal family) [29]. Let $\tilde{H}$ be a fuzzy soft inner product space. A family $\left\{\tilde{v}_{f_{i_{G\left(e_{i}\right)}}^{i}}\right\}$ of fuzzy soft elements of $\tilde{H}$ is called a fuzzy soft orthogonal family if

$$
\tilde{v}_{f_{i_{G\left(e_{i}\right)}}^{i}}^{i} \tilde{\perp}_{f_{j_{G\left(e_{j}\right)}}^{j}}^{j} ; i \neq j \text {, i.e., }\left\langle\tilde{v}_{f_{i_{G\left(e_{i}\right)}}^{i}, \tilde{v}_{f^{j_{G\left(e_{j}\right)}}}^{j}}\right\rangle \tilde{=} \tilde{0} ; i \neq j .
$$

Definition 28 (Fuzzy soft orthonormal family) [29]. Let $\tilde{H}$ be a fuzzy soft inner product space. A family $\left\{\tilde{v}_{f_{i_{G\left(e_{i}\right)}}^{i}}\right\}$ of fuzzy soft elements of $\tilde{H}$ is called a fuzzy soft orthonormal family if $\left\{\tilde{v}_{f_{i_{G\left(e_{i}\right)}}^{i}}\right\}$ is a fuzzy soft orthogonal family (i.e., satisfies the

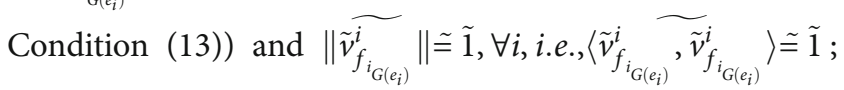
$i=j$.

Remark 29 (see [29]). Note that if $\left\{\tilde{v}_{f_{i_{G\left(e_{i}\right)}}^{i}}\right\}$ is a fuzzy soft orthonormal family, then $\left\langle\tilde{v}_{f_{i_{G\left(e_{i}\right)}}^{i}, \widetilde{v_{f^{j}}^{j}}}\right\rangle \tilde{\delta}_{j_{G\left(e_{j}\right)}}$, where

$$
\tilde{\delta}_{i j}=\left\{\begin{array}{l}
\tilde{1}, \text { if } i=j \\
\tilde{0}, \text { if } i \neq j
\end{array}\right.
$$

Example 30. The fuzzy soft set of fuzzy soft elements $(\tilde{j}, \tilde{\theta}, \tilde{\theta})$, $(\tilde{\theta}, \tilde{j}, \tilde{\theta})$ and $(\tilde{\theta}, \tilde{\theta}, \tilde{j})$ is a fuzzy soft orthonormal family in $\mathbb{R}^{3}(A)$.

Solution. Let $\tilde{e}_{1} \tilde{=}(\tilde{j}, \tilde{\theta}, \tilde{\theta}), \tilde{e}_{2} \tilde{=}(\tilde{\theta}, \tilde{j}, \tilde{\theta})$ and $\tilde{e}_{3} \tilde{=}(\tilde{\theta}, \tilde{\theta}, \tilde{j})$. It is clear that $\left\langle\tilde{e}_{i}, \tilde{e}_{j}\right\rangle \tilde{=} \tilde{\delta}_{i j} ; i, j=1,2,3$.

Example 31. The fuzzy soft set of fuzzy soft elements $(\tilde{j}, \tilde{\theta}$, $\tilde{\theta}, \tilde{\theta}, \cdots, \tilde{\theta}, \cdots),(\tilde{\theta}, \tilde{j}, \tilde{\theta}, \tilde{\theta}, \cdots, \tilde{\theta}, \cdots)$ and $(\tilde{\theta}, \tilde{\theta}, \tilde{j}, \tilde{\theta}, \tilde{\theta}, \cdots, \tilde{\theta}, \cdots), \cdots$ is a fuzzy soft orthonormal family in $\ell_{2}(A)$.

Solution. Let $\tilde{e}_{1} \tilde{=}(\tilde{j}, \tilde{\theta}, \tilde{\theta}, \tilde{\theta}, \cdots, \tilde{\theta}, \cdots), \quad \tilde{e}_{2} \tilde{=}(\tilde{\theta}, \tilde{j}, \tilde{\theta}, \tilde{\theta}, \cdots, \tilde{\theta}, \cdots)$ and $\tilde{e}_{3} \tilde{=}(\tilde{\theta}, \tilde{\theta}, \tilde{j}, \tilde{\theta}, \tilde{\theta}, \cdots, \tilde{\theta}), \cdots$. It is clear that $\left\langle\tilde{e}_{i}, \tilde{e}_{j}\right\rangle \tilde{=} \tilde{\delta}_{i j}$; $i$, $j=1,2,3, \cdots$. 
Example 32. Let $\tilde{H} \tilde{=} \mathscr{C}_{[\tilde{0}, \tilde{1}]}(A)$ be the space of all fuzzy soft complex-valued continuous functions on $[\tilde{0}, \tilde{1}]$. For $k=0, \pm$ $1, \pm 2, \cdots$, let $\left\{\tilde{e}_{k}\right\} \tilde{C} \tilde{H}$ be defined by:

$$
\tilde{e}_{k}(\tilde{x}) \tilde{=} \tilde{e}^{2 \pi i k \tilde{x}}, \tilde{x} \tilde{\epsilon}[\tilde{0}, \tilde{1}]
$$

Then, $\left\{\tilde{e}_{k}\right\}$ is a fuzzy soft orthonormal family in $\mathscr{C}_{[\tilde{0}, \tilde{1}]}(A)$.

Solution.

$$
\begin{aligned}
& \left.\left\langle\widetilde{\tilde{e}_{k}, \tilde{e}_{j}}\right\rangle=\int_{\tilde{0}}^{\tilde{1}} \tilde{e}_{k}(\tilde{x}) \tilde{e}_{j} \overline{(} \tilde{x}\right) d \tilde{x} \tilde{=} \int_{\tilde{0}}^{\tilde{1}} \tilde{e}^{2 \pi i k \tilde{x}} \tilde{e}^{-2 \pi i j \tilde{x}} d \tilde{x} \tilde{=} \int_{\tilde{0}}^{\tilde{1}} \tilde{e}^{2 \pi i(k-j) \tilde{x}} \\
& \cdot d \tilde{x}=\left\{\begin{array}{l}
\frac{\left[\tilde{e}^{2 \pi i(k-j) \tilde{x}}\right]_{\tilde{0}}^{\tilde{1}}}{2 \pi i(k-j)}, \text { if } k \neq j \\
\int_{\tilde{0}}^{\tilde{1}} d \tilde{x}, \text { if } k=j
\end{array}\right. \\
& =\left\{\begin{array}{l}
\frac{\left(\tilde{e}^{2 \pi i(k-j)}-\tilde{1}\right)}{2 \pi i(k-j)}, \text { if } k \neq j \\
\int_{\tilde{0}}^{\tilde{1}} d \tilde{x}, \text { if } k=j
\end{array}=\left\{\begin{array}{l}
\tilde{0}, \text { if } k \neq j \\
\tilde{1}, \text { if } k=j .
\end{array}\right.\right.
\end{aligned}
$$

Hence, $\left\{\tilde{e}_{k}\right\}$, where $\left\{\tilde{e}_{k}\right\}$ is defined as in (15), is a fuzzy soft orthonormal family in $\mathscr{C}_{[\tilde{0}, \tilde{1}]}(A)$.

Definition 33 (Fuzzy soft linear operator). Let $\tilde{T}: \operatorname{FSV}\left(\tilde{U}_{1}\right)$ $\rightarrow F S V\left(\tilde{U}_{2}\right)$ be an operator. Then $\tilde{T}$ is said to be fuzzy soft linear if

(1) $\tilde{T}\left(\tilde{v}_{f_{1_{G\left(e_{1}\right)}}}^{1}+\tilde{v}_{f_{2_{G\left(e_{2}\right)}}}^{2}\right) \tilde{=} \tilde{T}\left(\tilde{v}_{f_{1_{G\left(e_{1}\right)}}^{1}}^{1}\right)+\tilde{T}\left(\tilde{v}_{f_{2_{G\left(e_{2}\right)}}}^{2}\right)$, for all

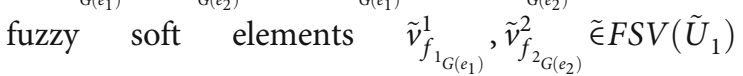
(additivity).

(2) $\tilde{T}\left(\tilde{c} \tilde{v}_{f_{G(e)}}\right) \tilde{=} \tilde{c} \tilde{T}\left(\tilde{v}_{f_{G(e)}}\right)$, for all fuzzy soft element $\tilde{v}_{f_{G(e)}}$ $\tilde{\epsilon} \operatorname{FSV}\left(\tilde{U}_{1}\right)$ and for all fuzzy soft scalar $\tilde{c}$ (homogeneity).

The Conditions $(1,2)$ can be combined in one condition as follows:

$$
\tilde{T}\left(\tilde{c}_{1} \tilde{v}_{f_{G\left(e_{1}\right)}}^{1}+\tilde{c}_{2} \tilde{v}_{f_{2_{G\left(e_{2}\right)}}}^{2}\right) \tilde{=} \tilde{c}_{1} \tilde{T}\left(\tilde{v}_{f_{1_{G\left(e_{1}\right)}}}^{1}\right)+\tilde{c}_{2} \tilde{T}\left(\tilde{v}_{f_{2_{G\left(e_{2}\right)}}^{2}}^{2}\right) \text {, for all }
$$
fuzzy soft elements $\tilde{v}_{f_{1_{G\left(e_{1}\right)}}^{1}}^{1}, \tilde{v}_{f_{2_{G\left(e_{2}\right)}}}^{2} \tilde{\epsilon} F S V\left(\tilde{U}_{1}\right)$ and for all fuzzy soft scalars $\tilde{c}_{1}, \tilde{c}_{2}$.

Definition 34 (Fuzzy soft linear operator in $\tilde{H}$ ). Let $\tilde{H}$ be a fuzzy soft Hilbert space. A fuzzy soft linear operator $\tilde{T}: \tilde{D}($ $\tilde{C} \tilde{H}) \longrightarrow \tilde{H}$ is called a fuzzy soft linear operator in $\tilde{H}$. If $\tilde{D}$ $\tilde{=} \tilde{H}$, then $\tilde{T}$ is a fuzzy soft linear operator on $\tilde{H}$, written $\tilde{T} \tilde{\epsilon}$ $\tilde{\mathbb{L}}(\tilde{H})$. If $\tilde{T}$ is fuzzy soft bounded (i.e., $\exists \tilde{k} \tilde{\in} \mathbb{R}^{+}(A)$ : $\left.\left\|\tilde{T}\left(\tilde{v}_{f_{G(e)}}\right)\right\| \tilde{\leq} \tilde{k}\left\|\tilde{v}_{f_{G(e)}}\right\| \forall \tilde{v}_{f_{G(e)}} \tilde{\epsilon} \tilde{H}\right)$, then $\tilde{T} \tilde{\epsilon} \tilde{\mathbb{B}}(\tilde{H})$.
Example 35. The fuzzy soft identity operator $\tilde{I}: \tilde{H} \longrightarrow \tilde{H}$ defined by $\tilde{I}\left(\tilde{v}_{f_{G(e)}}\right)=\tilde{v}_{f_{G(e)}}$, for all $\tilde{v}_{f_{G(e)}} \tilde{\epsilon} \tilde{H}$, is a fuzzy soft linear operator on $\tilde{H}$.

Example 36. The fuzzy soft zero operator $\tilde{0}: \tilde{H} \rightarrow \tilde{H}$ defined by $\tilde{0}\left(\tilde{v}_{f_{G(e)}}\right)=\tilde{\theta}$, for all $\tilde{v}_{f_{G(e)}} \tilde{\epsilon} \tilde{H}$, is a fuzzy soft linear operator on $\tilde{H}$.

Definition 37 (Fuzzy soft right shift operator). Let $\tilde{H}$ be a fuzzy soft Hilbert space. If $\tilde{v}_{f_{G(e)}} \tilde{=}\left(\tilde{v}_{f_{1_{G\left(e_{1}\right)}}^{1}}, \tilde{v}_{f_{2_{G\left(e_{2}\right)}}}^{2}, \tilde{v}_{f_{3_{G\left(e_{3}\right)}}}\right) \tilde{\epsilon} \tilde{H}$. Define the fuzzy soft operator $\tilde{R}$ as follows:

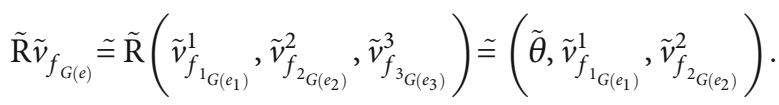

The fuzzy soft operator $\tilde{R}$ is called the fuzzy soft right shift operator.

Example 38. The fuzzy soft right shift operator $\tilde{R}: \tilde{H} \longrightarrow \tilde{H}$ defined as above in Definition (37) is a fuzzy soft linear operator on $\tilde{H}$.

Definition 39 (Fuzzy soft left shift operator). Let $\tilde{H}$ be a fuzzy

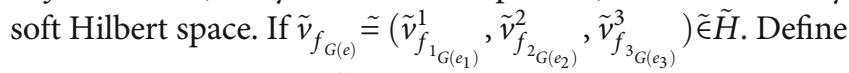
the fuzzy soft operator $\tilde{L}$ as follows:

$$
\tilde{\mathrm{L}} \tilde{v}_{f_{G(e)}} \tilde{=} \tilde{\mathrm{L}}\left(\tilde{v}_{f_{1_{G\left(e_{1}\right)}}^{1}}^{1}, \tilde{v}_{f_{{ } G\left(e_{2}\right)}}^{2}, \tilde{v}_{f_{3_{G\left(e_{3}\right)}}}^{3}\right) \tilde{=}\left(\tilde{v}_{f_{{ }^{G}\left(e_{2}\right)}}^{2}, \tilde{v}_{f^{3_{G\left(e_{3}\right)}}}^{3}, \tilde{\theta}\right) .
$$

The fuzzy soft operator $\tilde{\mathrm{L}}$ is called the fuzzy soft left shift operator.

Example 40. The fuzzy soft left shift operator $\tilde{L}: \tilde{H} \longrightarrow \tilde{H}$ defined as above in Definition 3.9 is a fuzzy soft linear operator on $\tilde{H}$.

Definition 41 (Fuzzy soft adjoint operator in $\tilde{H}$ ). The fuzzy soft adjoint operator $\tilde{T}^{*}$ of a fuzzy soft linear operator $\tilde{T}$ is defined by $\left\langle\tilde{T} \tilde{v}_{f_{1_{G\left(e_{1}\right)}}^{1}}, \tilde{v}_{f_{2_{G\left(e_{2}\right)}}^{2}}\right\rangle \tilde{=}\left\langle\tilde{v}_{f_{1_{G\left(e_{1}\right)}}^{1}}, \tilde{\tilde{T}}^{*} \tilde{v}_{f_{2_{G\left(e_{2}\right)}}^{2}}^{2}\right\rangle$, for all $\tilde{v}_{f_{1_{G\left(e_{1}\right)}} \tilde{\epsilon}}^{1} \tilde{H}$.

Example 42. The fuzzy soft left shift operator $\tilde{L}$ defined in Definition (39) is the fuzzy soft adjoint of the fuzzy soft right shift operator $\tilde{R}$ defined in Definition (37).

Example 43. Let $\tilde{H} \tilde{=} \ell_{2}(A)$ be the space of all fuzzy soft

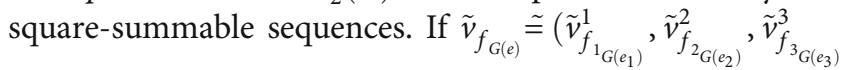
, ‥ $\tilde{\epsilon} \ell_{2}(A)$. By applying the fuzzy soft operator (17) from the above Definition (37), we can define the fuzzy soft right 
shift operator (the fuzzy soft unilateral shift operator) $\tilde{U}$ in $\ell_{2}(A)$ as follows:

$$
\begin{aligned}
\tilde{U} \tilde{v}_{f_{G(e)}} & \tilde{=} \tilde{U}\left(\tilde{v}_{f_{1_{G\left(e_{1}\right)}}^{1}}^{1}, \tilde{v}_{f_{2_{G\left(e_{2}\right)}}}^{2}, \tilde{v}_{f_{3_{G\left(e_{3}\right)}}^{3}}^{3}, \cdots\right) \\
& \approx\left(\tilde{\theta}, \tilde{v}_{f_{1_{G\left(e_{1}\right)}}^{1}}^{1}, \tilde{v}_{f_{2_{G\left(e_{2}\right)}}^{2}}^{2}, \tilde{v}_{f_{3_{G\left(e_{3}\right)}}^{3}}^{3}, \cdots\right) .
\end{aligned}
$$

If $\left\{\tilde{e}_{n}\right\}$ is a fuzzy soft orthonormal basis for $\ell_{2}(A)$, i.e., the set of fuzzy soft elements

$$
\begin{gathered}
\tilde{e}_{1} \tilde{=}(\tilde{j}, \tilde{\theta}, \tilde{\theta}, \tilde{\theta}, \cdots, \tilde{\theta}, \cdots), \\
\tilde{e}_{2} \tilde{=}(\tilde{\theta}, \tilde{j}, \tilde{\theta}, \tilde{\theta}, \cdots, \tilde{\theta}, \cdots), \\
\tilde{e}_{3} \tilde{=}(\tilde{\theta}, \tilde{\theta}, \tilde{j}, \tilde{\theta}, \tilde{\theta}, \cdots, \tilde{\theta}, \cdots),
\end{gathered}
$$

is a fuzzy soft orthonormal family (fuzzy soft orthonormal basis) in $\ell_{2}(A)$. Then, by using (19), we get:

$$
\tilde{U} \tilde{e}_{n} \tilde{=} \tilde{e}_{n+1}, n=1,2,3, \cdots \text {. }
$$

For the fuzzy soft adjoint $\tilde{U}^{\tilde{*}}$, we have:

$$
\left\langle\widetilde{\tilde{U}^{*} \tilde{e}_{i}}, \tilde{e}_{j}\right\rangle \tilde{=}\left\langle\tilde{e}_{i}, \widetilde{\tilde{U}} \tilde{e}_{j}\right\rangle \text {. }
$$

Therefore, by using Formula (21) in (22), the following is obtained:

$$
\left\langle\tilde{U}^{\tilde{*}} \tilde{e}_{i}, \tilde{e}_{j}\right\rangle \tilde{=}\left\langle\tilde{e}_{i}, \tilde{e}_{j+1}\right\rangle \tilde{=} \tilde{\delta}_{i, j+1}, i, j=1,2, \cdots
$$

Since

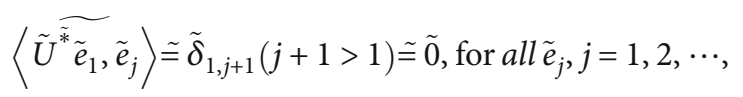

then

$$
\tilde{U}^{\tilde{*}} \tilde{e}_{1} \tilde{=} \tilde{\theta}
$$

If $i>1$, then we have:

$$
\tilde{\delta}_{i, j+1} \tilde{=} \tilde{\delta}_{i-1, j} \tilde{=}\left\langle\tilde{e}_{i-1}, \tilde{e}_{j}\right\rangle \text {. }
$$

Thus, by substituting from (26) in (23), we get:

$$
\left\langle\widetilde{\tilde{U}^{*}} \tilde{e}_{i}, \tilde{e}_{j}\right\rangle \tilde{=}\left\langle\widetilde{e_{i-1}, \tilde{e}_{j}}\right\rangle
$$

and therefore, the following formula is obtained:

$$
\tilde{U}^{\tilde{*}} \tilde{e}_{i} \tilde{e}_{i-1}, i=2,3, \cdots .
$$

Now, by applying (25) and (28), we have:

$$
\begin{aligned}
& \tilde{U}^{\tilde{*}} \tilde{v}_{f_{G(e)}} \tilde{=} \tilde{U}^{\tilde{*}}\left(\tilde{v}_{f_{1_{G\left(e_{1}\right)}}^{1}}, \tilde{v}_{f_{2_{G\left(e_{2}\right)}}^{2}}^{2}, \tilde{v}_{f_{3_{G\left(e_{3}\right)}}^{3}}, \cdots\right) \\
& \tilde{=} \tilde{U}^{\tilde{*}}\left(\tilde{v}_{f_{1_{G\left(e_{1}\right)}}^{1}} \tilde{e}_{1}+\tilde{v}_{f_{2_{G\left(e_{2}\right)}}^{2}}^{2} \tilde{e}_{2}+\tilde{v}_{f_{3_{G\left(e_{3}\right)}}^{3}} \tilde{e}_{3}+\cdots\right)
\end{aligned}
$$

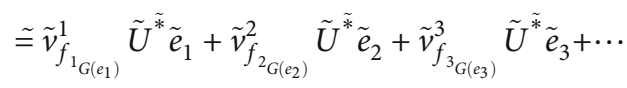

$$
\begin{aligned}
& \tilde{=} \tilde{v}_{f_{1_{G\left(e_{1}\right)}}^{1}}^{1} \times \tilde{\theta}+\tilde{v}_{f_{2_{G\left(e_{2}\right)}}^{2}} \tilde{e}_{1}+\tilde{v}_{f_{3_{G\left(e_{3}\right)}}^{3}}^{3} \tilde{e}_{2}+\cdots
\end{aligned}
$$

Then, by substituting from (20) in (29), we obtain:

$$
\begin{aligned}
& \tilde{U}^{\tilde{*}} \tilde{v}_{f_{G(e)}} \tilde{=} \tilde{v}_{f^{{ }_{G}\left(e_{2}\right)}}^{2}(\tilde{j}, \tilde{\theta}, \tilde{\theta}, \tilde{\theta}, \cdots, \tilde{\theta}, \cdots) \\
& +\tilde{v}_{f_{3_{G\left(e_{3}\right)}}^{3}}(\tilde{\theta}, \tilde{j}, \tilde{\theta}, \tilde{\theta}, \cdots, \tilde{\theta}, \cdots) \\
& +\cdots . \tilde{=}\left(\tilde{v}_{f_{{ }_{G\left(e_{2}\right)}}^{2}}, \tilde{v}_{f_{{ }_{G\left(e_{3}\right)}}^{3}}, \cdots\right) .
\end{aligned}
$$

Hence, we finally get:

$$
\begin{gathered}
\tilde{U}^{\tilde{*}} \tilde{v}_{f_{G(e)}} \tilde{=} \tilde{U}^{\tilde{*}}\left(\tilde{v}_{f_{1_{G\left(e_{1}\right)}}^{1},}, \tilde{v}_{f_{2_{G\left(e_{2}\right)}}^{2}}, \tilde{v}_{f_{3_{G\left(e_{3}\right)}}^{3}}, \cdots\right) \\
\tilde{=}\left(\tilde{v}_{f_{2_{G\left(e_{2}\right)}}^{2}}, \tilde{v}_{f_{3_{G\left(e_{3}\right)}}^{3}}, \ldots\right) .
\end{gathered}
$$

Equation (31) represents the fuzzy soft adjoint of fuzzy soft unilateral shift operator, which is usually said to be a fuzzy soft left shift operator.

Theorem 44. Let $\tilde{H}$ be a fuzzy soft inner product space and $\tilde{T} \tilde{\in} \tilde{\mathbb{L}}(\tilde{H})$. Then, we have $\tilde{\mathscr{R}}(\tilde{T})^{\tilde{\perp}} \tilde{=} \tilde{\mathcal{N}}\left(\tilde{T}^{*}\right)$, where $\tilde{\mathscr{R}}(\tilde{T})$ is the fuzzy soft range of $\tilde{T}$ and $\tilde{\mathcal{N}}\left(\tilde{T}^{*}\right)$ is the fuzzy soft null (fuzzy soft kernel) of $\tilde{T}^{*}$.

Proof. Let $\tilde{v}_{f_{1_{G\left(e_{1}\right)}}^{1}}^{1} \tilde{\epsilon} \tilde{H}$ and $\tilde{v}_{f_{2_{G\left(e_{2}\right)}}}^{2} \tilde{\epsilon} \tilde{\mathscr{R}}(\tilde{T})^{\tilde{\perp}}$. Then, we get

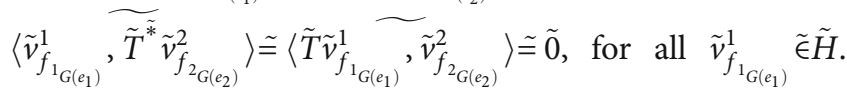
Therefore $\tilde{T}^{\tilde{*}} \tilde{v}_{f_{2_{G\left(e_{2}\right)}}^{2}} \approx \tilde{\theta}$, and thus $\tilde{v}_{f{ }_{G\left(e_{2}\right)}}^{2} \tilde{\epsilon} \tilde{\mathcal{N}}\left(\tilde{T}^{\tilde{*}}\right)$. That is to

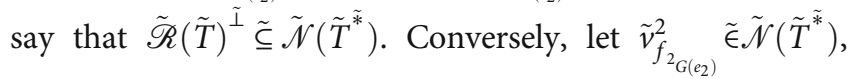

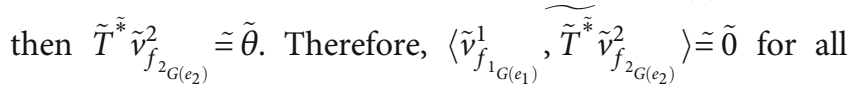
$\tilde{v}_{f_{1_{G\left(e_{1}\right)}}^{1}}^{1} \tilde{\epsilon} \tilde{H}$. But we have that $\left\langle\tilde{T} \tilde{v}_{f_{1_{G\left(e_{1}\right)}}^{1}}, \tilde{v}_{f_{2_{G\left(e_{2}\right)}}^{2}}^{2}\right\rangle \tilde{=}$

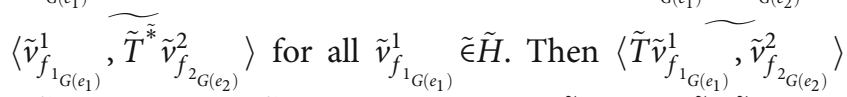
$\tilde{=} \tilde{0}$ for all $\tilde{v}_{f_{1_{G\left(e_{1}\right)}}^{1}}^{1} \tilde{\epsilon} \tilde{H}$. But we know that $\tilde{T} \tilde{v}_{f_{1_{G\left(e_{1}\right)}}^{1}}^{1} \tilde{\epsilon} \tilde{\mathscr{R}}(\tilde{T})$, then

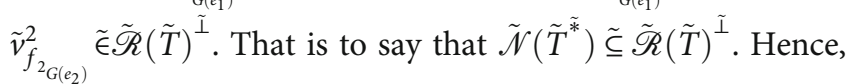
$\tilde{\mathscr{R}}(\tilde{T})^{\tilde{\perp}} \tilde{=} \tilde{\mathcal{N}}\left(\tilde{T}^{\tilde{*}}\right)$. 
Theorem 45. Let $\tilde{T}, \tilde{T}_{1}, \tilde{T}_{2} \tilde{\in} \tilde{\mathbb{B}}(\tilde{H})$, where $\tilde{H}$ is a fuzzy soft Hilbert space and let $\tilde{\lambda} \tilde{\epsilon} \mathbb{C}(A)$. Then, $\tilde{T}^{\tilde{*}^{\tilde{*}}} \cong \tilde{T},(\tilde{\lambda} \tilde{T}) \stackrel{\tilde{*}}{=} \tilde{\tilde{\lambda}} \tilde{T}^{\tilde{*}}$, $\left(\tilde{T}_{1}+\tilde{T}_{2}\right)^{\tilde{*}} \cong \tilde{T}_{1}^{\tilde{*}}+\tilde{T}_{2}^{\tilde{*}},\left(\tilde{T}_{1} \tilde{T}_{2}\right)^{\tilde{*}} \cong \tilde{T}_{2}^{\tilde{*}} \tilde{T}_{1}^{\tilde{*}}$, and if $\tilde{T}^{\tilde{-1}}$ exists, then $\left(\tilde{T}^{\tilde{*}}\right)^{\tilde{-1}}$ exists and $\left(\tilde{T}^{\tilde{-1}}\right)^{\tilde{*}} \tilde{=}\left(\tilde{T}^{\tilde{*}}\right)^{-\tilde{-1}}$.

Proof. To prove the first result, let $\tilde{v}_{f_{1_{G\left(e_{1}\right)}}^{1}}, \tilde{v}_{f_{L_{G\left(e_{2}\right)}}}^{2} \tilde{\epsilon} \tilde{H}$, then we have:

$$
\begin{aligned}
& \left\langle\tilde{T} \tilde{v}_{f_{1_{G\left(e_{1}\right)}}^{1}}, \tilde{v}_{f_{2_{G\left(e_{2}\right)}}^{2}}\right\rangle \tilde{=}\left\langle\tilde{v}_{f_{1_{G\left(e_{1}\right)}}^{1},}, \tilde{T}^{\tilde{v}^{*}} \tilde{v}_{f_{\mathrm{G}_{G\left(e_{2}\right)}}^{2}}\right\rangle
\end{aligned}
$$

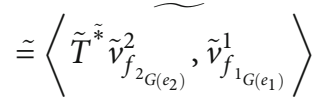

$$
\begin{aligned}
& \tilde{=\left\langle\tilde{v}_{f_{2_{G\left(e_{2}\right)}}^{2}}, \widetilde{\tilde{T}^{\tilde{*}^{*}}} \tilde{v}_{f_{1_{G\left(e_{1}\right)}}}\right\rangle} \\
& \tilde{=}\left\langle\tilde{T}^{\tilde{*}^{\tilde{*}}} \tilde{v}_{f_{1_{G\left(e_{1}\right)}}^{1}}, \tilde{v}_{f_{2_{G\left(e_{2}\right)}}^{2}}\right\rangle \text {. }
\end{aligned}
$$

To prove the last result, since $\left\langle\tilde{v}_{f_{1_{G\left(e_{1}\right)}}^{1}}, \widetilde{\tilde{I}^{\tilde{*}}} \tilde{v}_{f_{2_{G\left(e_{2}\right)}}^{2}}\right\rangle \tilde{=}$

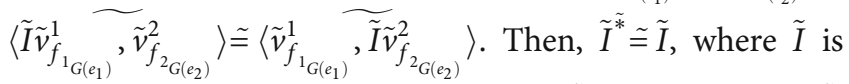
the fuzzy soft identity operator. Since $\tilde{T}^{\tilde{-1}}$ exists, then $\tilde{T} \tilde{T}^{-1}$ $\tilde{=} \tilde{I}$ and hence $\tilde{I} \tilde{I} \tilde{I}^{\tilde{*}} \tilde{=}\left(\tilde{T} \tilde{T}^{\tilde{-1}}\right)^{\tilde{*}} \tilde{=}\left(\tilde{T}^{\tilde{-1}}\right)^{\tilde{T}^{*}} \tilde{T}^{*}$. Therefore, $\left(\tilde{T}^{\tilde{*}}\right)^{\tilde{-1}}$ exists and $\left(\tilde{T}^{\tilde{*}}\right)^{\tilde{-1}} \tilde{=}\left(\tilde{T}^{-1}\right)^{\tilde{*}}$. The rest results can be proved similarly.

Theorem 46. Let $\tilde{T} \tilde{\epsilon} \tilde{\mathbb{L}}(\tilde{H})$, where $\tilde{H}$ is a fuzzy soft inner product space. If $\left\|\tilde{T}_{\tilde{v}_{f_{G(e)}}}\right\| \tilde{=}\left\|\widetilde{v}_{f_{G(e)}}\right\|$ for all $\tilde{v}_{f_{G(e)}} \tilde{\epsilon} \tilde{H}$, then

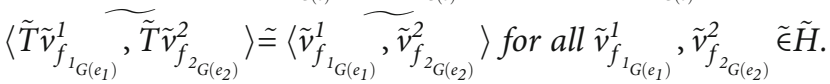

Proof. By using Theorem (22), we have:

$$
\begin{aligned}
& \left\langle\tilde{T} \tilde{v}_{f_{1_{G\left(e_{1}\right)}}^{1}} \sim \tilde{T} \tilde{v}_{f_{2_{G\left(e_{2}\right)}}^{2}}\right\rangle \tilde{=} \frac{\tilde{1}}{\tilde{4}}\left(\left\|\tilde{T} \tilde{v}_{f_{1_{G\left(e_{1}\right)}}^{1}}+\tilde{T} \tilde{v}_{f_{2_{G\left(e_{2}\right)}}^{2}}\right\|^{2}\right. \\
& -\left\|\tilde{T} \tilde{v}_{f_{1_{G\left(e_{1}\right)}}^{1}}-\tilde{T} \tilde{v}_{f_{\left.{ }_{G\left(e_{2}\right)}\right)}^{2}}\right\|^{2}+\tilde{i}\left\|\tilde{T} \tilde{v}_{f_{1_{G\left(e_{1}\right)}}^{1}}+\tilde{i} \tilde{T}_{v_{f_{G\left(e_{2}\right)}}^{2}}^{2}\right\|^{2} \\
& \left.-\tilde{i}\left\|\tilde{T} \tilde{v}_{f_{G_{G\left(e_{1}\right)}}^{1}}-\tilde{i} \tilde{T}_{f_{f_{G\left(e_{2}\right)}}^{2}}^{2}\right\|^{2}\right) \tilde{=} \tilde{\tilde{q}}\left(\left\|\tilde{T}\left(\tilde{v}_{f_{1_{G\left(e_{1}\right)}}^{1}}+\tilde{v}_{f_{{ }_{G\left(e_{2}\right)}}^{2}}\right)\right\|^{2}\right. \\
& -\left\|\tilde{T}\left(\tilde{v}_{f_{1_{G\left(e_{1}\right)}}^{1}}-\tilde{v}_{f_{{ }_{G\left(e_{2}\right)}}^{2}}^{2}\right)\right\|^{2}+\tilde{i}\left\|\tilde{T}\left(\tilde{v}_{f_{1_{G\left(e_{1}\right)}}^{1}}+\tilde{i}_{\tilde{v}_{f_{G\left(e_{2}\right)}}^{2}}^{2}\right)\right\|^{2} \\
& \left.-\tilde{i}\left\|\tilde{T}\left(\tilde{v}_{f_{\mathrm{G}_{\left(e_{1}\right)}}^{1}}-\tilde{i}_{\tilde{v}_{2_{G\left(e_{2}\right)}}^{2}}\right)\right\|^{2}\right) \text {. }
\end{aligned}
$$

Now, using the given that $\left\|\widetilde{T} \tilde{v}_{f_{G(e)}}\right\| \tilde{=}\left\|\widetilde{\tilde{v}_{f_{G(e)}}}\right\|$ for all $\tilde{v}_{f_{G(e)}}$ $\tilde{\epsilon} \tilde{H}$, we obtain that:

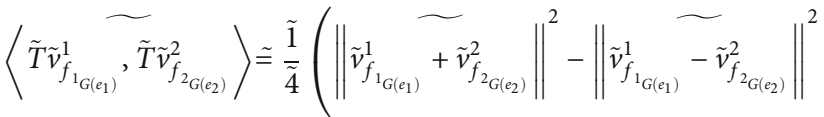

$$
\begin{aligned}
& \left.+\tilde{i}\left\|\tilde{v}_{f_{I_{G\left(e_{1}\right)}}^{1}}+\tilde{i}_{\tilde{v}_{f_{G\left(l_{2}\right)}}^{2}}^{2}\right\|^{2}-\tilde{i}\left\|\tilde{v}_{f_{I_{G\left(e_{1}\right)}}^{1}}-\tilde{i} \tilde{v}_{f_{L_{G\left(e_{2}\right)}}^{2}}\right\|^{2}\right)
\end{aligned}
$$

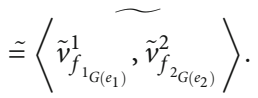

Theorem 47. Let $\tilde{T} \tilde{\epsilon} \tilde{\mathbb{B}}(\tilde{H})$, where $\tilde{H}$ is a fuzzy soft Hilbert space. Then, $\tilde{T}^{\tilde{*}}$ is fuzzy soft bounded and

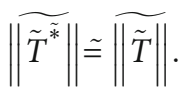

Proof. Using Theorem (17) and Theorem (16), we have:

$$
\begin{aligned}
& \left\|\tilde{T}^{\tilde{*} \tilde{v}_{f_{G(e)}}}\right\|^{2} \tilde{=}\left\langle\tilde{T}^{\tilde{*}} \tilde{v}_{f_{G(e)}} \widetilde{\tilde{T}^{\tilde{*}}} \tilde{v}_{f_{G(e)}}\right\rangle \tilde{=}\left\langle\tilde{T} \tilde{T}^{\tilde{*}} \tilde{v}_{f_{G(e)}}, \tilde{v}_{f_{G(e)}}\right\rangle \\
& \tilde{\leq}\left\|\tilde{T} \tilde{T}^{\tilde{*}} \tilde{v}_{f_{G(e)}}\right\|\left\|\widetilde{\tilde{v}_{f_{G(e)}}}\right\| \tilde{\leq}\|\tilde{T}\|\left\|\tilde{T}^{\tilde{*}} \tilde{v}_{f_{G(e)}}\right\|\left\|\widetilde{\tilde{v}_{f_{G(e)}}}\right\|,
\end{aligned}
$$

for all $\tilde{v}_{f_{G(e)}} \tilde{\epsilon} \tilde{H}$. Then $\left\|\tilde{T}^{\tilde{*} \tilde{v}_{f_{G(e)}}}\right\| \tilde{\leq}\|\widetilde{T}\|\left\|\widetilde{\tilde{v}_{f_{G(e)}}}\right\|$ for all $\tilde{v}_{f_{G(e)}}$ $\tilde{\epsilon} \tilde{H}$. Therefore,

$$
\left\|\widetilde{\tilde{T}^{\tilde{*}} \| \tilde{\leq}} \widetilde{\| \tilde{T}}\right\|
$$

and thus $\tilde{T}^{\tilde{*}}$ is fuzzy soft bounded. Similarly, using (1) from Theorem (45), we get:

$$
\widetilde{\| \tilde{T}}\|\tilde{=}\| \widetilde{\tilde{T}^{\tilde{*}^{*}}}\|\tilde{\leq}\| \widetilde{\tilde{T}^{\tilde{*}} \|}
$$

Hence, from (37), (38), we obtain that $\widetilde{\tilde{T}^{\tilde{T}}}\|\tilde{=}\| \widetilde{T} \|$.

Theorem 48. Let $\tilde{T} \tilde{\epsilon} \tilde{\mathbb{B}}(\tilde{H})$, where $\tilde{H}$ is a fuzzy soft Hilbert space. Then, $\left\|\widetilde{\tilde{T}^{\tilde{*}} \tilde{T}}\right\| \tilde{=}\|\widetilde{\tilde{T}}\| \stackrel{2}{=}\left\|\widetilde{\tilde{T}^{T^{*}}}\right\|$.

Proof. We have for all $\tilde{v}_{f_{G(e)}} \tilde{\epsilon} \tilde{H}$ :

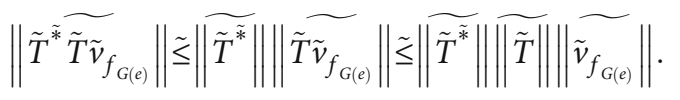

By using (35) from Theorem (47) in (39), we obtain that 
$\left\|\tilde{T}^{*} \widetilde{T}_{\tilde{v}_{f_{G(e)}}}\right\| \tilde{\leq}\|\widetilde{T}\|^{2}\left\|{\tilde{v_{f}}}_{f_{G(e)}}\right\|$ for all $\tilde{v}_{f_{G(e)}} \tilde{\epsilon} \tilde{H}$. Hence,

$$
\left\|\widetilde{\tilde{T}^{\tilde{T}} \tilde{T}}\right\| \tilde{\leq}\|\widetilde{T}\|^{2}
$$

Conversely, using Theorem (17), we get for all $\tilde{v}_{f_{G(e)}} \tilde{\epsilon} \tilde{H}$ :

$$
\begin{aligned}
\left\|\tilde{T} \tilde{v}_{f_{G(e)}}\right\|^{2} & \tilde{\langle}\left\langle\tilde{T} \tilde{v}_{f_{G(e)}} \widetilde{\tilde{T}} \tilde{v}_{f_{G(e)}}\right\rangle \tilde{=}\left\langle\tilde{v}_{f_{G(e)}}, \widetilde{\tilde{T}^{*} \tilde{T}} \tilde{v}_{f_{G(e)}}\right\rangle \\
& \tilde{\leq}\left\|\widetilde{\tilde{T}^{*} \tilde{T}}\right\|\left\|\tilde{v}_{f_{G(e)}}\right\|^{2} .
\end{aligned}
$$

Then, $\left\|\widetilde{T} \tilde{v}_{f_{G(e)}}\right\| \tilde{\leq}\left(\left\|\widetilde{\tilde{T}^{*}} \tilde{T}\right\|\right)^{1 / 2}\left\|\widetilde{\tilde{v}_{f_{G(e)}}}\right\|$ for all $\tilde{v}_{f_{G(e)}} \tilde{\epsilon} \tilde{H}$. Hence, $\|\widetilde{T}\| \tilde{\leq}\left(\left\|\widetilde{T}^{*} \tilde{T}\right\|\right)$. That is to say that

$$
\|\widetilde{T}\|^{2} \tilde{\leq}\left\|\widetilde{\tilde{T}^{*} \tilde{T}}\right\|
$$

Therefore, from (40), (42), the following result is obtained:

$$
\left\|\widetilde{\tilde{T}^{\tilde{*}} \tilde{T}}\right\| \tilde{=}\|\tilde{T}\|^{2}
$$

Now, by applying this result (43) for $\tilde{T}^{\tilde{*}}$ in place of $\tilde{T}$, we have $\left\|\widetilde{\tilde{T}^{\tilde{T}^{*}}}\right\| \tilde{=}\left\|\widetilde{T}^{\tilde{*}^{\tilde{*}}} \tilde{T}^{\tilde{*}}\right\| \tilde{=}\left\|{\widetilde{T^{*}}}^{2}\right\| \tilde{=}\|\widetilde{\widetilde{T}}\|^{2}$.

Definition 49 (Fuzzy soft resolvent set of $\tilde{T}$ ). Let $\tilde{T} \tilde{\in} \tilde{\mathbb{B}}(\tilde{H})$, where $\tilde{H}$ is a fuzzy soft Hilbert space. Then, we define $\tilde{\rho}(\tilde{T})$ $\tilde{=}\left\{\tilde{\lambda} \tilde{\epsilon} \mathbb{C}(A):(\tilde{\lambda} \tilde{I}-\tilde{T})^{\tilde{-1}}\right.$ exists and fuzzy soft bounded $\} \cong\{\tilde{\lambda}$ $\tilde{\epsilon} \mathbb{C}(A):|\tilde{\lambda}| \tilde{>}\|\tilde{\widetilde{T}}\|\}$.

We call $\tilde{\rho}(\tilde{T})$ the fuzzy soft resolvent set of a fuzzy soft linear operator $\tilde{T}$.

Definition 50 (Fuzzy soft spectrum of $\tilde{T}$ ). Let $\tilde{T} \tilde{\epsilon} \tilde{\mathbb{B}}(\tilde{H})$, where $\tilde{H}$ is a fuzzy soft Hilbert space. Then, we define $\tilde{\sigma}(\tilde{T}) \tilde{=} \tilde{\rho}$ $(\tilde{T})^{C} \tilde{=}\{\tilde{\lambda} \tilde{\epsilon} \mathbb{C}(A):|\tilde{\lambda}| \tilde{\leq}\|\tilde{T}\|\}$. We call $\tilde{\sigma}(\tilde{T})$ the fuzzy soft spectrum of a fuzzy soft linear operator $\tilde{T}$.

Definition 51 (Fuzzy soft spectral radius of $\tilde{T}$ ). Since, for $\tilde{T} \tilde{\epsilon}$ $\tilde{\mathbb{B}}(\tilde{H})$, where $\tilde{H}$ is a fuzzy soft Hilbert space, $\tilde{\sigma}(\tilde{T})$ is nonempty fuzzy soft compact (fuzzy soft closed and fuzzy soft bounded), we define $\tilde{r}_{\tilde{\sigma}}(\tilde{T}) \tilde{=} \sup \{|\tilde{\lambda}|: \tilde{\lambda} \tilde{\epsilon} \tilde{\sigma}(\tilde{T})\}$. We call $\tilde{r}_{\tilde{\sigma}}($ $\tilde{T})$ the fuzzy soft spectral radius of a fuzzy soft linear operator $\tilde{T}$. Also, we can show that $\tilde{r}_{\tilde{\sigma}}(\tilde{T})=\lim _{n \rightarrow \infty}\left\|\widetilde{T}^{n}\right\|^{1 / n}$.

Definition 52 (Fuzzy soft point spectrum of $\tilde{T}) . \tilde{\lambda} \tilde{\epsilon} \mathbb{C}(A)$ is said to be a fuzzy soft eigenvalue of a fuzzy soft linear operator $\tilde{T}$ if $\tilde{\lambda} \tilde{I}-\tilde{T}$ is not fuzzy soft injective, i.e., there exists a non-zero fuzzy soft element $\tilde{v}_{f_{G(e)}} \tilde{\epsilon} \tilde{H}$ such that $\tilde{T} \tilde{v}_{f_{G(e)}} \tilde{=} \tilde{\lambda}$ $\tilde{v}_{f_{G(e)}}$. Moreover, $\tilde{v}_{f_{G(e)}} \tilde{\neq} \tilde{\theta}$ is called the fuzzy soft eigenvector of a fuzzy soft linear operator $\tilde{T}$ corresponding to $\tilde{\lambda}$. The set of all such $\tilde{\lambda}$ is called the fuzzy soft point spectrum of a fuzzy soft linear operator $\tilde{T}$, denoted by $\tilde{\sigma}_{p}(\tilde{T})$. We have $\tilde{\sigma}_{p}$ $(\tilde{T}) \tilde{C} \tilde{\sigma}(\tilde{T})$.

Definition 53 (Fuzzy soft approximate point spectrum of $\tilde{T}$ ). $\tilde{\lambda} \tilde{\epsilon} \mathbb{C}(A)$ is said to be a fuzzy soft approximate eigenvalue of a fuzzy soft linear operator $\tilde{T}$ if there exists a fuzzy soft sequence of fuzzy soft elements $\left\{\tilde{v}_{f_{n_{G\left(e_{n}\right)}}^{n}}\right\}$ in $\tilde{H}$ such that $\left\|\tilde{v}_{f_{n_{G}\left(e_{n}\right)}^{n}}^{n}\right\| \tilde{=} \tilde{1}$ and $\tilde{T} \tilde{v}_{f_{n_{G}\left(e_{n}\right)}^{n}}^{n}-\tilde{\lambda} \tilde{v}_{f_{n_{G\left(e_{n}\right)}}^{n}} \longrightarrow \tilde{0}$. The set of all such $\tilde{\lambda}$ is called the fuzzy soft approximate point spectrum of a fuzzy soft linear operator $\tilde{T}$, denoted by $\tilde{\sigma}_{a}(\tilde{T})$. We have $\tilde{\sigma}_{a}$ $(\tilde{T}) \tilde{C} \tilde{\sigma}(\tilde{T})$.

Definition 54 (Fuzzy soft comparison spectrum of $\tilde{T}$ ). The set of all $\tilde{\lambda} \tilde{\in} \mathbb{C}(A)$ such that the fuzzy soft range of $\tilde{\lambda} \tilde{I}-\tilde{T}$ is not fuzzy soft dense in $\tilde{H}$, (i.e. $\tilde{\mathscr{R}}(\tilde{\tilde{\lambda}} \tilde{I} \tilde{T}) \tilde{\neq} \tilde{H})$ is called the fuzzy soft comparison spectrum of a fuzzy soft linear operator $\tilde{T}$, denoted by $\tilde{\sigma}_{\text {Com }}(\tilde{T})$.

Definition 55 (Fuzzy soft residual spectrum of $\tilde{T}$ ). The set of all $\tilde{\lambda} \tilde{\epsilon} \mathbb{C}(A)$ such that $\tilde{\lambda} \tilde{I}-\tilde{T}$ is fuzzy soft injective, but its fuzzy soft range is not fuzzy soft dense in $\tilde{H}$ is called the fuzzy soft residual spectrum of a fuzzy soft linear operator $\tilde{T}$, denoted by $\tilde{\sigma}_{r}(\tilde{T})$. That is to say that:

$$
\tilde{\sigma}_{r}(\tilde{T}) \tilde{=} \tilde{\sigma}_{\operatorname{Com}}(\tilde{T}) \backslash \tilde{\sigma}_{p}(\tilde{T}) .
$$

Definition 56 (Fuzzy soft continuous spectrum of $\tilde{T}$ ). The set of all $\tilde{\lambda} \tilde{\in} \mathbb{C}(A)$ such that $\tilde{\lambda} \tilde{I}-\tilde{T}$ is fuzzy soft injective and has fuzzy soft dense range in $\tilde{H}$, but is fuzzy soft singular (has no fuzzy soft inverse) is called the fuzzy soft continuous spectrum of a fuzzy soft linear operator $\tilde{T}$, denoted by $\tilde{\sigma}_{c}(\tilde{T})$. It is clear that $\tilde{\sigma}_{p}(\tilde{T}) \tilde{\cup} \tilde{\sigma}_{c}(\tilde{T}) \tilde{C} \tilde{\sigma}_{a}(\tilde{T})$, and

$$
\tilde{\sigma}(\tilde{T}) \tilde{=} \tilde{\sigma}_{p}(\tilde{T}) \tilde{\cup} \tilde{\sigma}_{c}(\tilde{T}) \tilde{\cup} \tilde{\sigma}_{r}(\tilde{T})
$$

the terms on the right being mutually disjoint.

Theorem 57. Let $\tilde{H}$ be a fuzzy soft Hilbert space and $\tilde{T} \tilde{\epsilon} \tilde{\mathbb{B}}(\tilde{H})$. If $\tilde{\lambda} \tilde{\epsilon} \tilde{\sigma}_{a}(\tilde{T})$, then, $|\tilde{\lambda}| \tilde{\leq}|| \tilde{\tilde{T}} \|$.

Proof. Let $\tilde{\lambda} \tilde{\epsilon} \tilde{\sigma}_{a}(\tilde{T})$, then there exists a fuzzy soft sequence of fuzzy soft elements $\left\{\tilde{v}_{f_{n_{G}\left(e_{n}\right)}^{n}}\right\}$ in $\tilde{H}$ such that $\left\|\tilde{v}_{f_{n_{G\left(e_{n}\right)}}^{n}}\right\| \tilde{=} \tilde{1}$ and

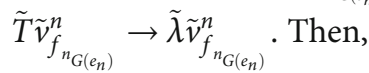


$\left\|\tilde{T}{\widetilde{v_{f}}}_{f_{n_{G\left(e_{n}\right)}}^{n}}\right\| \longrightarrow \widetilde{\tilde{\lambda}} \tilde{v}_{f_{n_{G\left(e_{n}\right)}^{n}}^{n}} \| \tilde{=}|\tilde{\lambda}|$, i.e., $\lim _{n \rightarrow \infty}\left\|\tilde{T} \tilde{v}_{f_{n_{G\left(e_{n}\right)}}^{n}}\right\| \tilde{=}|\tilde{\lambda}|$.

Therefore,

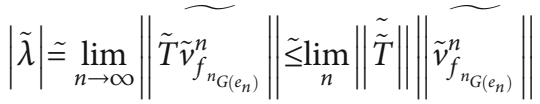

$$
\begin{aligned}
& \tilde{=}\|\tilde{\tilde{T}}\| \lim _{n}\left\|\widetilde{\tilde{v}_{f_{n_{G\left(e_{n}\right)}}^{n}}}\right\| \tilde{=}\|\tilde{\tilde{T}}\| .
\end{aligned}
$$

Corollary 58. Let $\tilde{H}$ be a fuzzy soft Hilbert space and $\tilde{T} \tilde{\epsilon} \tilde{\mathbb{B}}(\tilde{H})$ . If $\tilde{\lambda} \tilde{\epsilon} \tilde{\sigma}_{p}(\tilde{T})$, then, $|\tilde{\lambda}| \tilde{\leq}\|\tilde{\widetilde{T}}\|$.

Proof. From (45), we get $\tilde{\sigma}_{p}(\tilde{T}) \tilde{C} \tilde{\sigma}_{a}(\tilde{T})$. Then, Theorem (57) completes the proof.

Theorem 59. If $\tilde{T} \tilde{\epsilon} \tilde{\mathbb{B}}(\tilde{H})$, where $\tilde{H}$ is a fuzzy soft Hilbert space. Then,

(1) $\tilde{T}$ and $\tilde{T}^{\tilde{*}}$ have the same fuzzy soft resolvent set, i.e., $\tilde{\rho}(\tilde{T}) \tilde{=} \tilde{\rho}\left(\tilde{T}^{\tilde{*}}\right)$.

(2) $\tilde{T}$ and $\tilde{T}^{\tilde{*}}$ have the same fuzzy soft spectrum, i.e., $\tilde{\sigma}(\tilde{T}) \tilde{=} \tilde{\sigma}\left(\tilde{T}^{\tilde{*}}\right)$.

(3) $\tilde{\sigma}_{r}(\tilde{T}) \tilde{C} \tilde{\sigma}_{p}\left(\tilde{T}^{\tilde{*}}\right)$ and $\tilde{\sigma}_{p}(\tilde{T}) \tilde{C} \tilde{\sigma}_{p}\left(\tilde{T}^{\tilde{*}}\right) \tilde{\cup} \tilde{\sigma}_{r}(\tilde{T})$.

Theorem 60. The fuzzy soft point spectrum of fuzzy soft right shift operator on fuzzy soft square-summable sequences space $\ell_{2}(A)$ has no fuzzy soft eigenvalues, i.e.,

$$
\tilde{\sigma}_{p}(\tilde{U}) \tilde{=} \phi
$$

Proof. Let $\tilde{\lambda} \tilde{\epsilon} \tilde{\sigma}_{p}(\tilde{U})$, then there exists $\tilde{\theta} \tilde{\neq} \tilde{v}_{f_{G(e)}} \tilde{=}\left(\tilde{v}_{f_{1_{G\left(e_{1}\right)}}^{1}}\right.$, $\left.\tilde{v}_{f_{G\left(e_{2}\right)}}^{2}, \tilde{v}_{f_{B_{G\left(e_{3}\right)}}}, \cdots\right) \tilde{\epsilon} \ell_{2}(A)$ such that $\tilde{U}_{f_{G(e)}} \approx \tilde{\lambda} \tilde{v}_{f_{G(e)}}$.

Therefore, by using (19), we obtain:

$$
\begin{aligned}
& \left(\tilde{\theta}, \tilde{v}_{f_{1_{G\left(e_{1}\right)}}^{1}}, \tilde{v}_{f_{{ }_{G\left(e_{2}\right)}}^{2}}^{2}, \tilde{v}_{f_{B_{G\left(e_{3}\right)}}^{3}}, \ldots\right) \\
& \quad \tilde{=}\left(\tilde{\lambda} \tilde{v}_{f_{1_{G\left(e_{1}\right)}}^{1}}, \tilde{\lambda} \tilde{v}_{f_{{ }_{G\left(e_{2}\right)}}^{2}}, \tilde{\lambda} \tilde{v}_{f_{3_{G\left(e_{3}\right)}}^{3}}, \ldots\right) .
\end{aligned}
$$

Then, from (48), we get that $\tilde{\lambda} \tilde{v}_{f_{1_{G\left(e_{1}\right)}}^{1}} \approx \tilde{\theta}$ and $\tilde{v}_{f_{{ }_{G\left(e_{n}\right)}}^{n}} \simeq \tilde{\lambda}$ $\tilde{v}_{f_{n+1} G\left(e_{n+1}\right)}^{n+1}$ for all $n$.

We have two cases:

(1) If $\tilde{\lambda} \tilde{=} \tilde{0}$, then $\tilde{v}_{f_{n_{G\left(e_{n}\right)}}^{n}} \tilde{=} \tilde{\theta}$ for all $n$ and hence $\tilde{v}_{f_{G(e)}} \tilde{=} \tilde{\theta}$.
(2) If $\tilde{\lambda} \tilde{\neq} \tilde{0}$, then $\tilde{v}_{f_{1_{G\left(e_{1}\right)}}^{1}}=\tilde{\theta}$ and $\tilde{\lambda} \tilde{v}_{f_{G\left(e_{2}\right)}}^{2} \tilde{=} \tilde{\theta}$, then $\tilde{v}_{f_{2_{G\left(e_{2}\right)}}^{2}}$ $\tilde{=} \tilde{\theta}, \cdots$, and so on. Therefore, we obtain that $\tilde{v}_{f_{n_{G}\left(e_{n}\right)}^{n}}$ $\tilde{=} \tilde{\theta}$ for all $n$ and hence $\tilde{v}_{f_{G(e)}} \tilde{=} \tilde{\theta}$.

Now, each case of $(1,2)$ implies that $\tilde{v}_{f_{G(e)}} \tilde{\theta}$, but this is a contradiction with $\tilde{v}_{f_{G(e)}} \tilde{\neq} \tilde{\theta}$ assumed above at first. Hence, $\tilde{\sigma}_{p}(\tilde{U})$ must be empty, i.e., $\tilde{\sigma}_{p}(\tilde{U}) \tilde{=} \phi$, which is required.

Corollary 61. The fuzzy soft residual spectrum of fuzzy soft right shift operator is equal to the fuzzy soft comparison spectrum of it on fuzzy soft square-summable sequences space $\ell_{2}$ (A), i.e.,

$$
\tilde{\sigma}_{r}(\tilde{U}) \tilde{=} \tilde{\sigma}_{\operatorname{Com}}(\tilde{U}) .
$$

Proof. The proof is straight forward by substituting from (47) in (44) and hence (49) is true.

Theorem 62. The fuzzy soft point spectrum of fuzzy soft left shift operator on fuzzy soft square-summable sequences space $\ell_{2}(A)$ is the fuzzy soft open disk $|\tilde{\lambda}| \tilde{<} \tilde{1}$, i.e.,

$$
\tilde{\sigma}_{p}\left(\tilde{U}^{\tilde{*}}\right) \tilde{=}(\tilde{\lambda} \tilde{\epsilon} \mathbb{C}(A):|\tilde{\lambda}| \tilde{<} \tilde{1}) .
$$

Proof. Let $\tilde{\lambda} \tilde{\epsilon} \tilde{\sigma}_{p}\left(\tilde{U}^{\tilde{*}}\right)$, then there exists $\tilde{\theta} \tilde{\neq} \tilde{v}_{f_{G(e)}} \tilde{=}\left(\tilde{v}_{f_{\mathrm{G}_{G\left(e_{1}\right)}}^{1}}\right.$,

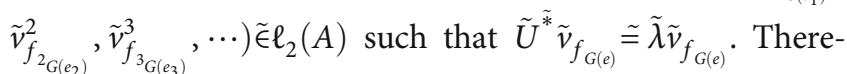
fore, by using (19), we obtain:

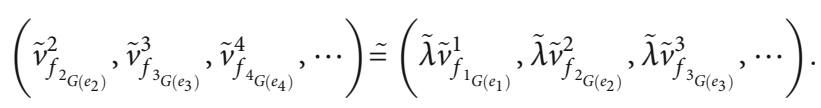

Then, from (51), we get that $\tilde{v}_{f_{n+1}{ }^{n\left(e_{n+1}\right)}}^{n+1} \cong \tilde{\lambda} \tilde{v}_{f_{n_{G\left(e_{n}\right)}}^{n}}$ for all $n$. That is to say that,

$$
\tilde{v}_{f_{\mathrm{G}_{G\left(e_{2}\right)}}^{2}} \tilde{=} \tilde{\lambda}_{f_{1_{G\left(e_{1}\right)}}^{1}}
$$

and

$$
\tilde{v}_{f_{\mathrm{G}_{G\left(e_{3}\right)}}^{3}} \tilde{=} \tilde{\lambda}_{f_{2_{G\left(e_{2}\right)}}^{2}}^{2} .
$$

Therefore, by substituting for $\tilde{v}_{f_{G\left(e_{2}\right)}}^{2}$ from (52) in (53), we have:

$$
\tilde{v}_{f_{G_{G\left(e_{3}\right)}}} \approx \tilde{\lambda}\left(\tilde{\lambda} \tilde{v}_{f_{1_{G\left(e_{1}\right)}}^{1}}\right) \tilde{=} \tilde{\lambda}^{2} \tilde{v}_{f_{1_{G\left(e_{1}\right)}}^{1}} .
$$

In addition, we can repeat this process for

$$
\tilde{v}_{f^{4_{G\left(e_{4}\right)}}}^{4} \tilde{=} \tilde{\lambda} \tilde{v}_{f_{3_{G\left(e_{3}\right)}}^{3}}
$$


by substituting for $\tilde{v}_{f_{3_{G\left(e_{3}\right)}}^{3}}$ from (54) in (55) as follows:

$$
\tilde{v}_{f_{4_{G\left(e_{4}\right)}}}^{4} \tilde{=} \tilde{\lambda}\left(\tilde{\lambda}^{2} \tilde{v}_{f_{1_{G\left(e_{1}\right)}}}^{1}\right)=\tilde{\lambda}^{3} \tilde{v}_{f_{1_{G\left(e_{1}\right)}}^{1}}
$$

and so on. Thus, we get that:

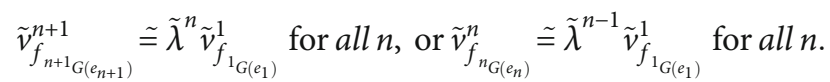

Now, we have two cases:

(1) If $\tilde{v}_{f_{1_{G\left(e_{1}\right)}}} \stackrel{=}{=}$, then $\tilde{v}_{f_{n_{G\left(e_{n}\right)}}^{n}} \cong \tilde{\theta}$ for all $n$ and hence $\tilde{v}_{f_{G(e)}}$ $\tilde{=} \tilde{\theta}$, which is a contradiction with $\tilde{v}_{f_{G(e)}} \tilde{\neq} \tilde{\theta}$ assumed above at first.

(2) If $\quad \tilde{v}_{f_{1_{G\left(e_{1}\right)}}}^{1} \tilde{\neq} \tilde{\theta}, \quad$ then $\quad \sum_{n=1}^{\infty}\left\|\tilde{v}_{f_{n_{G\left(e_{n}\right)}}^{n}}^{\sim}\right\|^{2} \tilde{=} \sum_{n=1}^{\infty}$ $\left\|\tilde{\lambda}^{n-1} \tilde{v}_{f_{1_{G\left(e_{1}\right)}}^{1}}\right\| \stackrel{2}{=} \sum_{n=1}^{\infty}|\tilde{\lambda}|^{2 n-2}\left\|\tilde{v}_{f_{G\left(e_{1}\right)}^{1}}^{\sim}\right\|^{2}$, and therefore | $\tilde{\lambda} \mid$ must be less than $\tilde{1}$ for this fuzzy soft geometric series to be convergent. Hence, $\left\|\tilde{v}_{f_{n_{G\left(e_{n}\right)}}^{n}}^{\sim}\right\| \tilde{<} \infty$ if and only if $|\tilde{\lambda}| \tilde{<} \tilde{1}$.

And finally this proves that (50) is satisfied.

Theorem 63. The fuzzy soft conjugate space $\tilde{H}^{\tilde{*}}$ of any fuzzy soft Hilbert space $\tilde{H}$ is the same as $\tilde{H}$, i.e. $\tilde{H}^{*} \tilde{=} \tilde{H}(\tilde{H}$ and $\tilde{H}^{*}$ are fuzzy soft isomorphic).

Proof. Firstly, we want to prove that every fuzzy soft element $\tilde{w}_{h_{G(e)}} \tilde{\epsilon} \tilde{H}$ defines a fuzzy soft linear continuous functional $\tilde{\tilde{\epsilon}} \tilde{\epsilon}$ $\tilde{H}^{\tilde{*}}$ with the same fuzzy soft norm (i.e., to prove that $\|\tilde{\tilde{\tilde{F}}}\| \tilde{\leq}$ $\left.\left\|\widetilde{\tilde{w}_{h_{G(e)}}}\right\|\right)$. Let $\tilde{w}_{h_{G(e)}} \tilde{\epsilon} \tilde{H}$ and define $\tilde{\tilde{f}} \tilde{\epsilon} \tilde{H}^{\tilde{*}}$ as follows $\tilde{\mathfrak{f}}\left(\tilde{v}_{f_{G(a)}}\right) \tilde{=}$ $\left\langle\tilde{v}_{f_{G(a)}}, \tilde{w}_{h_{G(e)}}\right\rangle$. To prove that $\tilde{\mathfrak{f}}$ is fuzzy soft linear, let $\tilde{v}_{f_{G(a)}}$, $\tilde{u}_{g_{G(b)}} \tilde{\epsilon} \tilde{H}$ and let $\tilde{\lambda}, \tilde{\mu}$ be fuzzy soft scalars. Then, we have:

$$
\begin{aligned}
& \tilde{\mathfrak{f}}\left(\tilde{\lambda} \tilde{v}_{f_{G(a)}}+\tilde{\mu} \tilde{u}_{g_{G(b)}}\right) \tilde{=}\left\langle\tilde{\lambda} \tilde{v}_{f_{G(a)}}+\widetilde{\tilde{\mu}} \tilde{u}_{g_{G(b)}}, \tilde{w}_{h_{G(e)}}\right\rangle \\
& \tilde{=}\left\langle\tilde{\lambda} \tilde{v}_{f_{G(a)}}, \tilde{w}_{h_{G(e)}}\right\rangle+\left\langle\tilde{\mu} \tilde{u}_{g_{G(b)}}, \tilde{w}_{h_{G(e)}}\right\rangle \tilde{=} \tilde{\lambda}\left\langle\tilde{v}_{f_{G(a)}} \widetilde{\tilde{w}_{h_{G(e)}}}\right\rangle \\
& +\tilde{\mu}\left\langle\tilde{u}_{g_{G(b)}}, \tilde{w}_{h_{G(e)}}\right\rangle \tilde{=} \tilde{\lambda} \tilde{\mathfrak{f}}\left(\tilde{v}_{f_{G(a)}}\right)+\tilde{\mu} \tilde{\mathfrak{F}}\left(\tilde{u}_{g_{G(b)}}\right) \text {. }
\end{aligned}
$$
get:

Now, by using fuzzy soft Cauchy-Schwartz inequality, we

$$
\left|\tilde{\mathfrak{f}}\left(\tilde{v}_{f_{G(a)}}\right)\right| \tilde{=}\left|\left\langle\tilde{v}_{f_{G(a)}}, \tilde{w}_{h_{G(e)}}\right\rangle\right| \tilde{\leq}|| \tilde{v}_{f_{G(a)}}^{\sim}\|\| \tilde{w}_{h_{G(e)}} \| .
$$

But, we have:

$$
\widetilde{\| \tilde{\mathfrak{f}}} \| \tilde{=} \sup _{\tilde{v}_{f_{G(a)}}} \frac{\left|\tilde{\mathrm{f}}\left(\tilde{v}_{f_{G(a)}}\right)\right|}{\left\|\tilde{v}_{f_{G(a)}}\right\|} .
$$

Therefore, by substituting from (59) in (60), we obtain

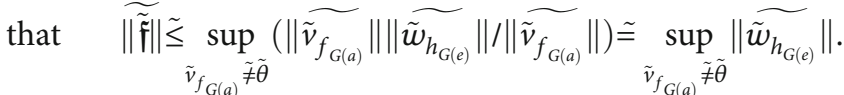
That is to say that,

$$
\tilde{v}_{f_{G(a)} \tilde{\neq} \tilde{\theta}} \tilde{G(a)} \tilde{v}_{f_{G(a)} \tilde{f} \tilde{\theta}}
$$

$$
\|\widetilde{\tilde{f}}\| \tilde{\leq}\left\|\widetilde{\tilde{w}_{h_{G(e)}}}\right\| .
$$

Conversely, we want to prove that every fuzzy soft linear continuous functional $\tilde{\tilde{f}} \tilde{\in} \tilde{H}^{*}$ defines a unique fuzzy soft element $\tilde{w}_{h_{G(e)}} \tilde{\epsilon} \tilde{H}$ with the same fuzzy soft norm (i.e., to prove that $\left.\left\|\widetilde{\tilde{w}_{h_{G(e)}}}\right\| \tilde{\leq}\|\widetilde{\tilde{f}}\|\right)$. Let $\tilde{\mathfrak{f}} \tilde{\epsilon} \tilde{H}^{*}$ be a fuzzy soft linear continuous functional on $\tilde{H}$, and let $\tilde{\mathfrak{B}} \tilde{=} \operatorname{ker} \tilde{\mathfrak{f}} \tilde{=}\left\{\tilde{v}_{f_{G(a)}} \tilde{\in} \tilde{H}: \tilde{\mathfrak{f}}\left(\tilde{v}_{f_{G(a)}}\right) \tilde{=} \tilde{\theta}\right\}$. To prove that $\tilde{\mathfrak{B}}$ is a fuzzy soft subspace of $\tilde{H}$, we have first to prove that $\tilde{\mathfrak{B}}$ is a fuzzy soft linear manifold of $\tilde{H}$ and second we have to prove that $\tilde{\mathfrak{B}}$ is fuzzy soft closed. First, to prove that $\tilde{\mathfrak{B}}$ is a fuzzy soft linear manifold of $\tilde{H}$, let $\tilde{v}_{f_{1_{G\left(a_{1}\right)}}^{1}}$, $\tilde{v}_{f_{2_{G\left(a_{2}\right)}}}^{2} \tilde{\epsilon} \tilde{\mathfrak{B}}$ and let $\tilde{\alpha}, \tilde{\beta}$ be fuzzy soft scalars. Then, $\tilde{\mathfrak{f}}\left(\tilde{v}_{f_{1_{G\left(a_{1}\right)}}^{1}}\right)$

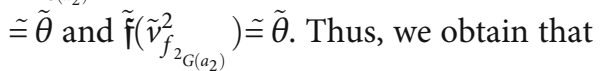

$$
\begin{gathered}
\tilde{\mathfrak{f}}\left(\tilde{\alpha} \tilde{v}_{f_{1_{G\left(a_{1}\right)}}^{1}}^{1}+\tilde{\beta} \tilde{v}_{f_{2_{G\left(a_{2}\right)}}^{2}}^{2}\right) \tilde{=} \tilde{\mathfrak{f}}\left(\tilde{\alpha} \tilde{v}_{f_{1_{G\left(a_{1}\right)}}^{1}}\right)+\tilde{\mathfrak{f}}\left(\tilde{\beta} \tilde{v}_{f_{2_{G\left(a_{2}\right)}}^{2}}\right) \\
\quad \tilde{\alpha} \tilde{\hat{\mathfrak{f}}}\left(\tilde{v}_{f_{1_{G\left(a_{1}\right)}}^{1}}^{1}\right)+\tilde{\beta} \tilde{\mathfrak{f}}\left(\tilde{v}_{f_{2_{G\left(a_{2}\right)}}^{2}}^{2}\right) \tilde{\alpha} \tilde{\alpha} \cdot \tilde{\theta}+\tilde{\beta} \cdot \tilde{\theta} \tilde{=} \tilde{\theta} .
\end{gathered}
$$

Therefore, we have $\tilde{\alpha} \tilde{v}_{f_{1_{G\left(a_{1}\right)}}^{1}}+\tilde{\beta} \tilde{v}_{f G\left(a_{2}\right)}^{2} \tilde{\epsilon} \tilde{\mathfrak{B}}$. Hence, $\tilde{\mathfrak{B}}$ is a fuzzy soft linear manifold of $\tilde{H}$. Second, to prove that $\tilde{\mathfrak{B}}$ is

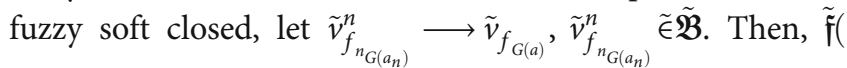
$\left.\tilde{v}_{\left.f_{n_{G\left(a_{n}\right)}}^{n}\right)}\right) \tilde{\theta}$. But $\tilde{\mathfrak{f}}$ is fuzzy soft continuous, then $\tilde{\mathfrak{f}}\left(\tilde{v}_{\left.f_{n_{G\left(a_{n}\right)}}^{n}\right)}\right)$ $\longrightarrow \tilde{\mathfrak{f}}\left(\tilde{v}_{f_{G(a)}}\right)$. Thus, $\tilde{\mathfrak{f}}\left(\tilde{v}_{f_{G(a)}}\right) \tilde{=} \tilde{\theta}$. Therefore, $\tilde{v}_{f_{G(a)}} \tilde{\in} \tilde{\mathfrak{B}}$ and hence $\tilde{\mathfrak{B}}$ is fuzzy soft closed. That is to say that, $\tilde{\mathfrak{B}}$ is a fuzzy soft subspace of $\tilde{H}$. Then, $\tilde{H} \tilde{=} \tilde{\mathfrak{B}} \tilde{\oplus} \tilde{\mathfrak{B}}^{\tilde{\perp}}$. Therefore, every fuzzy soft element $\tilde{v}_{f_{G(a)}} \tilde{\epsilon} \tilde{H}$ can be written uniquely as $\tilde{v}_{f_{G(a)}} \tilde{=} \tilde{u}_{g_{G(b)}}+\tilde{z}_{q_{G(d)}}$, $\tilde{u}_{g_{G(b)}} \tilde{\in} \tilde{\mathfrak{B}}, \tilde{z}_{q_{G(d)}} \tilde{\perp} \tilde{\mathfrak{B}}$. Then, $\tilde{\mathfrak{f}}\left(\tilde{u}_{g_{G(b)}}\right) \tilde{=}$ $\tilde{\theta}$, but $\tilde{\mathfrak{f}}\left(\tilde{z}_{q_{G(d)}}\right) \tilde{\neq} \tilde{\theta}$. Let $\tilde{v}_{f_{G(a)}} \tilde{\notin} \tilde{\mathfrak{B}}$ (i.e., $\left.\tilde{v}_{f_{G(a)}} \tilde{\epsilon} \tilde{H}-\tilde{\mathfrak{B}}\right)$, then $\tilde{v}_{f_{G(a)}} \tilde{=} \tilde{u}_{g_{G(b)}}+\tilde{z}_{q_{G(d)}}$, where $\tilde{z}_{q_{G(d)}} \tilde{\neq} \tilde{\theta}$ and $\tilde{\mathfrak{f}}\left(\tilde{z}_{q_{G(d)}}\right) \tilde{=} \tilde{\eta} \tilde{\neq} \tilde{0}$. Let 
$\tilde{v}_{f_{1_{G\left(a_{1}\right)}}^{1}} \tilde{=}\left(\tilde{z}_{q_{G(d)}} / \tilde{\eta}\right)$. Therefore

$$
\tilde{\mathfrak{f}}\left(\tilde{v}_{f_{1_{G\left(a_{1}\right)}}^{1}}\right) \tilde{=} \frac{\tilde{\mathfrak{f}}\left(\tilde{z}_{q_{G(d)}}\right)}{\tilde{\eta}} \tilde{=} \frac{\tilde{\eta}}{\tilde{\eta}} \tilde{=} \tilde{1}
$$

If $\tilde{v}_{f_{G(a)}}$ is any fuzzy soft point of $\tilde{H}$, then

$$
\tilde{\mathfrak{f}}\left(\tilde{v}_{f_{G(a)}}\right) \tilde{=} \tilde{\xi} \tilde{=} \tilde{\xi} \cdot \tilde{1} .
$$

Thus, by substituting from (63) in (64), we obtain that

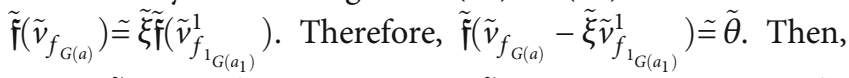
$\tilde{v}_{f_{G(a)}}-\tilde{\xi} \tilde{v}_{f_{1_{G\left(a_{1}\right)}}^{1}} \tilde{\epsilon} \tilde{\mathfrak{B}}$. Let $\quad \tilde{v}_{f_{G(a)}}-\tilde{\xi} \tilde{v}_{f_{1_{G\left(a_{1}\right)}}^{1}}=\tilde{u}_{g_{G(b)}}, \quad \tilde{u}_{g_{G(b)}} \tilde{\epsilon} \tilde{\mathfrak{B}}$. Hence, $\tilde{v}_{f_{G(a)}} \tilde{=} \tilde{u}_{g_{G(b)}}+\tilde{\xi} \tilde{v}_{f_{1_{G\left(a_{1}\right)}}^{1}}, \tilde{u}_{g_{G(b)}} \tilde{\epsilon} \tilde{\mathfrak{B}}, \tilde{\xi} \tilde{v}_{f_{1_{G\left(a_{1}\right)}}^{1}}^{1} \tilde{\perp} \tilde{\mathfrak{B}}$. Therefore, we have:

$$
\begin{aligned}
& \left\langle\tilde{v}_{f_{G(a)}}, \widetilde{v_{f_{1_{G\left(a_{1}\right)}}}^{1}}\right\rangle \tilde{=}\left\langle\tilde{u}_{g_{G(b)}}+\widetilde{\xi} \tilde{v}_{f_{1_{G\left(a_{1}\right)}}^{1}}, \tilde{v}_{f_{1_{G\left(a_{1}\right)}}^{1}}\right\rangle
\end{aligned}
$$

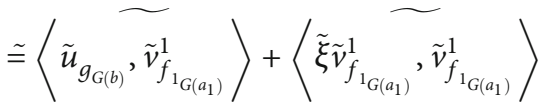

$$
\begin{aligned}
& \tilde{=} \tilde{0}+\tilde{\xi}\left\langle\tilde{v}_{f_{1_{G\left(a_{1}\right)}}^{1}}, \tilde{v}_{f_{1_{G\left(a_{1}\right)}}}^{1}\right\rangle \tilde{=} \tilde{\xi}\left\|\tilde{v}_{f_{1_{G\left(a_{1}\right)}}^{1}}\right\|^{2} .
\end{aligned}
$$

Then, $\left\langle\tilde{v}_{f_{G(a)}},\left(\tilde{v}_{f_{1_{G\left(a_{1}\right)}}^{1}} /\left\|\tilde{v}_{f_{1_{G\left(a_{1}\right)}}^{1}}\right\|^{2}\right)\right\rangle \tilde{=} \tilde{\xi} \tilde{=} \tilde{\mathfrak{f}}\left(\tilde{v}_{f_{G(a)}}\right)$. Let $\tilde{w}_{h_{G(e)}}$ $\tilde{=}\left(\tilde{v}_{f_{1_{G\left(a_{1}\right)}}^{1}} /\left\|\widetilde{v}_{f_{1_{G\left(a_{1}\right)}}^{1}}\right\|^{2}\right) \tilde{\epsilon} \tilde{H}$, then $\|\widetilde{\tilde{F}}\| \tilde{=} \sup _{\tilde{v}_{f_{G(a)}} \tilde{f} \tilde{\theta}}\left(\left|\tilde{\tilde{f}}\left(\tilde{v}_{f_{G(a)}}\right)\right| /\left\|\widetilde{\tilde{v}_{f_{G(a)}}}\right\|\right.$

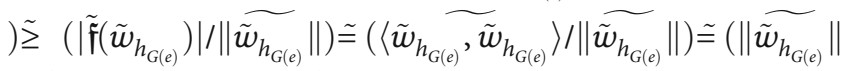
$\left.\left\|\widetilde{\tilde{w}_{h_{G(e)}}}\right\| /\left\|\widetilde{\tilde{w}_{h_{G(e)}}}\right\|\right) \tilde{=}\left\|\widetilde{\tilde{w}_{h_{G(e)}}}\right\|$. That is to say that,

$$
\left\|\widetilde{\tilde{w}_{h_{G(e)}}}\right\| \tilde{\leq}\|\tilde{\tilde{\tilde{f}}}\|
$$

Hence, from (61) and (66), we obtain that $\|\tilde{\tilde{\tilde{r}}}\| \tilde{=}$ $\left\|\widetilde{\tilde{w}_{h_{G(e)}}}\right\|$.

At the end, we have to show that the fuzzy soft element $\tilde{w}_{h_{G(e)}}$ is unique. Suppose that

$$
\begin{aligned}
& \tilde{\mathfrak{f}}\left(\tilde{v}_{f_{G(a)}}\right) \tilde{=}\left\langle\tilde{v}_{f_{G(a)}}, \tilde{w}_{h_{G(e)}}\right\rangle, \forall \tilde{v}_{f_{G(a)}} \tilde{\epsilon} \tilde{H} . \Longrightarrow\left\langle\tilde{v}_{f_{G(a)}} \widetilde{\tilde{w}_{h_{G(e)}}}\right\rangle \\
& \tilde{=}\left\langle\tilde{v}_{f_{G(a)}}, \tilde{w}_{h_{G\left(e^{c}\right)}}\right\rangle, \forall \tilde{v}_{f_{G(a)}} \tilde{\epsilon} \tilde{H} . \Longrightarrow\left\langle\tilde{v}_{f_{G(a)}}, \tilde{w}_{h_{G(e)}}-\tilde{w}_{h_{G\left(e^{\prime}\right)}}\right\rangle \\
& \tilde{=} \tilde{0}, \forall \tilde{v}_{f_{G(a)}} \tilde{\epsilon} \tilde{H} . \Longrightarrow \tilde{w}_{h_{G(e)}}-\tilde{w}_{h_{G\left(e^{\prime}\right)}^{\prime}} \tilde{=} \tilde{\theta} . \Longrightarrow \tilde{w}_{h_{G(e)}} \tilde{\sim}_{w_{G\left(e^{e}\right)}} \text {. }
\end{aligned}
$$

\section{Conclusions and Future Work}

The fuzzy version or the soft version of topics like metric spaces, normed spaces, Banach spaces, operators, dual spaces, functionals, inner product spaces, Hilbert spaces, operators on Hilbert spaces and many other topics have been studied by many mathematicians. On the other hand, combining fuzzy and soft sets together is not only more general than using only one of them but also gives us more extended and accurate results. Few researchers have studied some of those general extensions concepts such as fuzzy soft normed spaces and fuzzy soft metric spaces. We have defined the fuzzy soft inner product space $(\tilde{U}, \widetilde{\langle\cdot, \cdot\rangle})$ by using the concept of fuzzy soft vector $\tilde{v}_{f_{G(e)}}$ modified in our work and have shown that $\mathbb{C}^{n}(A), \mathbb{R}^{n}(A), \ell_{2}(A)$ and $\mathscr{C}_{[\tilde{0}, \tilde{1}]}(A)$ are suitable examples of fuzzy soft inner product spaces. Also, the definition of the fuzzy soft Hilbert space $\tilde{H}$ with $\mathbb{C}^{n}(A), \mathbb{R}^{n}(A)$ and $\ell_{2}(A)$ as examples of it have been introduced. In addition, fuzzy soft linear operator $\tilde{T}$ in fuzzy soft Hilbert space $\tilde{H}$ has been investigated. Moreover, the fuzzy soft orthogonal family and the fuzzy soft orthonormal family with examples on them have been established and some related theorems, examples and many more results of fuzzy soft linear operators with their proofs have been introduced. Furthermore, fuzzy soft right shift operator and fuzzy soft left shift operator with examples, on $\ell_{2}(A)$, have been investigated. In addition, we have defined the fuzzy soft resolvent set, the fuzzy soft spectrum, the fuzzy soft spectral radius, the fuzzy soft point spectrum, the fuzzy soft approximate point spectrum, the fuzzy soft comparison spectrum, the fuzzy soft residual spectrum and the fuzzy soft continuous spectrum of the fuzzy soft linear operators and we have given the relations between them. Moreover, it has been shown that, on $\ell_{2}(A)$, the fuzzy soft point spectrum of fuzzy soft right shift operator has no fuzzy soft eigenvalues, the fuzzy soft residual spectrum of fuzzy soft right shift operator is equal to the fuzzy soft comparison spectrum of it and the fuzzy soft point spectrum of fuzzy soft left shift operator is the fuzzy soft open disk $|\tilde{\lambda}| \tilde{<}$ $\tilde{1}$. To make the picture complete, we have proved that the fuzzy soft Hilbert space has the fuzzy soft self-duality property. Our results can be considered as generalizations for the well-known (crisp) ones. This type of investigations fills some gaps in the literature. The authors can introduce new results by using similar techniques in this paper. Some special types of fuzzy soft linear operators in fuzzy soft Hilbert spaces will be studied in further investigations depending on the definition of the fuzzy soft linear operator in fuzzy soft Hilbert space given in this paper. Finally, there are still many topics to study by applying the fuzzy soft notion on them.

\section{Data Availability}

Not applicable.

\section{Conflicts of Interest}

The authors declare that they have no conflict of interest. 


\section{Acknowledgments}

The authors would like to thank the referees for their valuable suggestions and comments.

\section{References}

[1] L. A. Zadeh, "Fuzzy sets," Information and Control, vol. 8, no. 3, pp. 338-353, 1965.

[2] D. Molodtsov, "Soft set theory-First results," Computers \& Mathematcs with Applications, vol. 37, no. 4-5, pp. 19-31, 1999.

[3] S. Bayramov and C. Gunduz, "Soft locally compact spaces and soft paracompact spaces," Journal of Mathematics and System, vol. 3, pp. 122-130, 2013.

[4] S. Das and S. K. Samanta, "Soft Metric," Annals of Fuzzy Mathematics and Informatics, vol. 6, no. 1, pp. 77-94, 2013.

[5] M. I. Yazar, T. Bilgin, S. Bayramov, and C. Gunduz (Aras), "A new view on soft normed spaces," International Mathematical Forum, vol. 9, no. 24, pp. 1149-1159, 2014.

[6] S. Das and S. K. Samanta, "On soft inner product spaces," Annals of Fuzzy Mathematics and Informatics, vol. 6, no. 1, pp. 151-170, 2013.

[7] M. I. Yazar, C. G. Aras, and S. Bayramov, "Results on soft Hilbert spaces," TWMS Journal of Applied and Engineering Mathematics, vol. 9, no. 1, pp. 159-164, 2019.

[8] M. Tareq Al-Shami, "Investigation and corrigendum to some results related to g-soft equality and gf -soft equality relations," Filomat, vol. 33, no. 11, pp. 3375-3383, 2019.

[9] T. M. Al-shami and M. E. El-Shafei, "Partial belong relation on soft separation axioms and decision-making problem: two birds with one stone," Soft Computing, vol. 24, no. 7, pp. 5377-5387, 2020.

[10] T. M. Al-shami, M. E. El-Shafei, and M. Abo-Elhamayel, "On soft topological ordered spaces," Journal of King Saud Universiy Science, vol. 31, no. 4, pp. 556-566, 2019.

[11] M. E. El-Shafei, M. Abo-Elhamayel, and T. M. Al-shami, "Partial soft separation axioms and soft compact spaces," Filomat, vol. 32, no. 13, pp. 4755-4771, 2018.

[12] M. E. El-Shafei and T. M. Al-shami, "Applications of partial belong and total non-belong relations on soft separation axioms and decision-making problem," Computational and Applied Mathematics, vol. 39, no. 3, 2020.

[13] P. K. Maji, R. Biswas, and A. R. Roy, "Fuzzy soft set," Journal of Fuzzy Mathematics, vol. 9, no. 3, pp. 677-692, 2001.

[14] T. J. Neog, D. K. Sut, and G. C. Hazarika, "Fuzzy soft topological spaces," International Journal of Latest Trends in Mathematics, vol. 2, no. 1, pp. 54-67, 2012.

[15] C. Gunaseeli, Some Contributions to Special Fuzzy Topological Spaces, [Ph.D. Thesis], Bharathidasan University, Tiruchirappalli, India, 2012.

[16] T. Beaula and C. Gunaseeli, "On fuzzy soft metric spaces," Malaya Journal of Matematik, vol. 2, no. 3, pp. 197-202, 2014.

[17] T. Beaula and M. M. Priyanga, "A new notion for fuzzy soft normed linear space," International Journal of Fuzzy Mathematical Archive, vol. 9, no. 1, pp. 81-90, 2015.

[18] M. R. Hashmi, M. Riaz, and F. Smarandache, "M-polar neutrosophic topology with applications to multi-criteria decisionmaking in medical diagnosis and clustering analysis," International Journal of Fuzzy Systems, vol. 22, no. 1, pp. 273-292, 2020.
[19] K. Naeem, M. Riaz, X. Peng, and D. Afzal, "Pythagorean fuzzy soft MCGDM methods based on TOPSIS, VIKOR and aggregation operators." Journal of Intelligent \& Fuzzy Systems, vol. 37, no. 5, pp. 6937-6957, 2019.

[20] M. Riaz, N. Çağman, I. Zareef, and M. Aslam, "N-soft topology and its applications to multi-criteria group decision-making," Journal of Intelligent \& Fuzzy Systems, vol. 36, no. 6, pp. 6521-6536, 2019.

[21] M. Riaz and M. R. Hashmi, "Linear diophantine fuzzy set and its applications towards multi-attribute decision-making problems," Journal of Intelligent \& Fuzzy Systems, vol. 37, no. 4, pp. 5417-5439, 2019.

[22] M. Riaz and S. T. Tehrim, "Bipolar fuzzy soft mappings with application to bipolar disorders," International Journal of Biomathematics, vol. 12, no. 7, p. 1950080, 2019.

[23] M. Riaz and S. T. Tehrim, "Cubic bipolar fuzzy ordered weighted geometric aggregation operators and their application using internal and external cubic bipolar fuzzy data," Computational and Applied Mathematics, vol. 38, no. 2, 2019.

[24] M. Riaz and S. T. Tehrim, "Multi-attribute group decisionmaking based on cubic bipolar fuzzy information using averaging aggregation operators," Journal of Intelligent \& Fuzzy Systems, vol. 37, no. 2, pp. 2473-2494, 2019.

[25] S. T. Tehrim and M. Riaz, "A novel extension of TOPSIS to MCGDM with bipolar Neutrosophic soft topology," Journal of Intelligent \& Fuzzy Systems, vol. 37, no. 4, pp. 5531-5549, 2019.

[26] P. K. Maji, R. Biswas, and A. R. Roy, "Soft set theory," Computers \& Mathematcs with Applications, vol. 45, no. 4-5, pp. 555-562, 2003.

[27] J. Mahanta and P. K. Das, "Fuzzy soft topological spaces," Journal of Intelligent Fuzzy Systems, vol. 32, pp. 443-450, 2017.

[28] N. Faried, M. S. S. Ali, and H. H. Sakr, "Fuzzy soft inner product spaces," Applied Mathematics \& Information Sciences, vol. 14, no. 4, 2020.

[29] N. Faried, M. S. S. Ali, and H. H. Sakr, "Fuzzy soft Hilbert spaces," Mathematics and Statistics, vol. 8, no. 3, 2020. 